bioRxiv preprint doi: https://doi.org/10.1101/2020.03.22.002634; this version posted May 30, 2020. The copyright holder for this preprint (which was not certified by peer review) is the author/funder. All rights reserved. No reuse allowed without permission.

Dynamic Normalization

May 30, 2020

\title{
Dynamic Normalization
}

David J. Heeger*,1,2 and Klavdia O. Zemlianova²

Department of Psychology ${ }^{1}$ and Center for Neural Science ${ }^{2}$

New York University

4 Washington Place, room 621

New York, NY 10003

*: Corresponding author

Email: david.heeger@nyu.edu

ORCID ID: 0000-0002-3282-9898

Classification. Biological Sciences: Neuroscience; Social Sciences: Psychological and Cognitive Sciences.

Keywords: computational neuroscience, recurrent neural network, V1, normalization, gamma oscillation

Abstract

The normalization model has been applied to explain neural activity in diverse neural systems including primary visual cortex (V1). The model's defining characteristic is that the response of each neuron is divided by a factor that includes a weighted sum of activity of a pool of neurons. In spite of the success of the normalization model, there are 3 unresolved issues. 1) Experimental evidence supports the hypothesis that normalization in V1 operates via recurrent amplification, i.e., amplifying weak inputs more than strong inputs. It is unknown how normalization arises from recurrent amplification. 2) Experiments have demonstrated that normalization is weighted such that each weight specifies how one neuron contributes to another's normalization pool. It is unknown how weighted normalization arises from a recurrent circuit. 3) Neural activity in V1 exhibits complex dynamics, including gamma oscillations, linked to normalization. It is unknown how these dynamics emerge from normalization. Here, a new family of recurrent circuit models is reported, each of which comprises coupled neural integrators to implement normalization via recurrent amplification with arbitrary normalization weights, some of which can recapitulate key experimental observations of the dynamics of neural activity in V1.

\section{Significance Statement}

A family of recurrent circuit models is proposed to explain the dynamics of neural activity in primary visual cortex (V1). Each of the models in this family exhibits steady state output responses that are already known to fit a wide range of experimental data from diverse neural systems. These models can recapitulate the complex dynamics of $\mathrm{V} 1$ activity, including oscillations (so-called gamma oscillations, $\sim 30-80 \mathrm{~Hz}$ ). This theoretical framework may also be used to explain key aspects of working memory and motor control. Consequently, the same circuit architecture is applicable to a variety of neural systems, and V1 can be used as a model system for understanding the neural computations in many brain areas. 
bioRxiv preprint doi: https://doi.org/10.1101/2020.03.22.002634; this version posted May 30, 2020. The copyright holder for this preprint (which was not certified by peer review) is the author/funder. All rights reserved. No reuse allowed without permission.

Dynamic Normalization

May 30, 2020

\section{Introduction}

The normalization model was initially developed to explain stimulus-evoked responses of neurons in primary visual cortex (V1) (1-7), but has since been applied to explain neural activity and behavior in diverse cognitive processes and neural systems (8-52). The defining characteristic of normalization is that the response of each neuron is divided by a factor that includes a weighted sum of activity of a pool of neurons (Fig. 1a). In V1, this normalization pool includes neurons selective for different visual stimulus features and spatial positions (i.e., receptive-field locations).

The normalization model mimics many well-documented physiological phenomena in V1 $(29,53-63)$ and their perceptual analogues (64-68). 1) Responses saturate (level off) when increasing the contrast of a preferred orientation test stimulus (e.g., a grating restricted to a neuron's receptive field, RF) (Fig. 1b, blue curve). 2) Responses to a non-preferred orientation are smaller than responses to the preferred orientation by a constant scale factor at all contrasts, in spite of response saturation (Fig. 1b, red vs. blue curves). Response saturation, consequently, is due to a network effect rather than a mechanism that is intrinsic to each neuron individually (e.g., refractory period) because responses saturate at the same contrast, not the same firing rate, for preferred and non-preferred stimuli. 3) Responses to two or more stimuli presented together are much less than the linear sum of the individual responses. Cross-orientation suppression results when a mask stimulus (e.g., a grating of fixed contrast) that is orthogonal to the preferred orientation is superimposed with a preferred-orientation test stimulus (Fig. 1c, yellow vs. blue curves). Likewise, surround suppression results when a mask stimulus is added in the region surrounding a neuron's RF. Different stimuli suppress responses by different amounts, suggesting the normalization is "tuned" or "weighted" $(14,55,60,61,69-72)$. The normalization weights specify the contribution of one neuron to another's normalization pool, thereby determining the tuning of normalization.

Normalization has been shown to serve a number of functions in a variety of neural systems (73) including automatic gain control (needed because of limited dynamic range) $(4,8,74)$, simplifying read-out $(8,75,76)$, conferring invariance with respect to one or more stimulus dimensions (e.g., contrast, odorant concentration) $(4,8$, $28,74,77)$, switching between averaging vs. winner-take-all (19), contributing to decorrelation \& statistical independence of neural responses $(10,28,78,79)$, stabilizing delay-period activity $(80)$, and facilitating learning $(81$, 82).

Neural activity in $\mathrm{V} 1$ exhibits complex dynamics linked to normalization. The rate of response increase following stimulus onset is typically faster than the rate of response decrease following stimulus offset (83). The rate of response increase is also stimulus dependent: faster for stimuli placed in the center of a RF and slower on the flanks of the RF (83). The timing of response suppression depends on its strength (72). Temporal-frequency tuning depends on stimulus contrast, and simple-cell response phase depends on contrast $(6,55,84-86)$. Complex dynamics are evident also in the combined activity (e.g., as measured with local field potentials, LFPs) of populations of neurons. LFPs exhibit so-called gamma oscillations $(\sim 30-80 \mathrm{~Hz})$ that have also been linked to normalization $(35,87,88)$. Oscillation amplitude and frequency depend systematically on stimulus contrast, size, and spatial pattern $(35,87,89-102)$.

The circuit mechanisms underlying normalization are not well understood. Experimental evidence supports the hypothesis that normalization operates via recurrent amplification, i.e., amplifying weak inputs more than
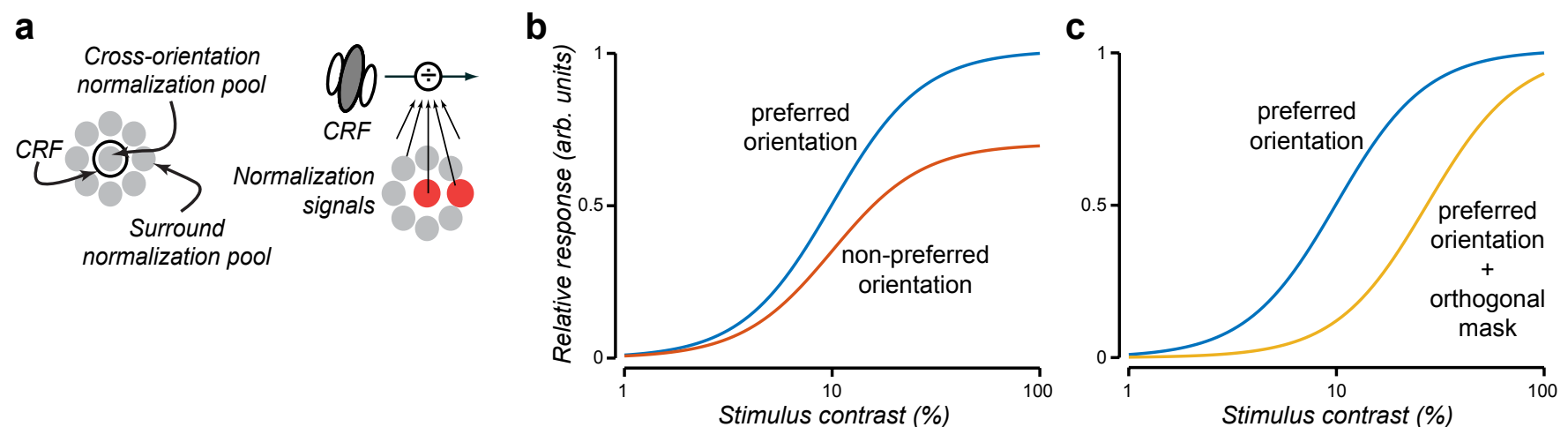

Figure 1. Normalization model. a. Conceptual diagram of normalization in which the neuron's response is suppressed by a weighted sum of activity of a pool of neurons. b. Response saturation. Blue, responses to preferred stimulus orientation saturate at high contrasts. Orange, responses to non-preferred orientation are a scaled-down copy of the responses to preferred orientation, saturating at the same contrast. c. Cross-orientation suppression. Blue, preferred orientation. Yellow, superimposing an orthogonal stimulus (fixed contrast) suppresses responses to the preferred orientation. A similar result would be observed by adding a stimulus component that surrounds the preferred stimulus (surround suppression). 
bioRxiv preprint doi: https://doi.org/10.1101/2020.03.22.002634; this version posted May 30, 2020. The copyright holder for this preprint (which was not certified by peer review) is the author/funder. All rights reserved. No reuse allowed without permission.

Dynamic Normalization

May 30, 2020

strong inputs (103-106). The recurrent amplification hypothesis is also supported by anatomy: cortical circuits are dominated by recurrent connections (107-112). We have known since we first introduced the normalization model that it can be implemented in a recurrent circuit $(4,5)$. Since then, several hypotheses for the circuit mechanisms underlying normalization have been proposed, including shunting inhibition, synaptic depression, and inhibitionstabilized networks $(6,39,55,113-117)$. See also Refs. (118-120) for precursors to these circuit models. But these models do not rely on recurrent amplification to achieve normalization and/or they do not exhibit complex dynamics (including gamma oscillations) linked to normalization (see Discussion). Furthermore, these previous models only approximate weighted normalization; this has practical consequences for making experimentallytestable predictions and for fitting data (see Discussion).

Here, we introduce and characterize a family of dynamical systems that implement normalization with recurrent amplification. When the input drive is constant over time, each of the recurrent circuits in this family exhibits output responses that follow the normalization equation exactly, with arbitrary (non-negative) normalization weights. Each model in this family is expressed as a coupled system of neural integrators, composed of two classes of neurons: principal cells and modulator cells. The key idea is that the amount of recurrent amplification in the principal cells depends inversely on the responses of the modulator cells. When the input is weak, the modulator cells have small responses and there is a large amount of recurrent amplification. When the input is strong, the modulator cell responses are large, which shuts down the recurrent amplification. The various models in this family of dynamical systems imply different circuits, some (but not all) of which recapitulate the complex dynamics V1 activity, including gamma oscillations. Although we focus on V1, this family of models is applicable to many neural systems (see Discussion).

A preliminary version of this work was posted on a preprint server (121). MATLAB code for recreating the simulation results is available at http://hdl.handle.net/2451/61045 (122).

\section{Results}

\section{Recurrent circuit models of normalization}

We begin by introducing one model out of the family of dynamical systems that implement normalization with recurrent amplification. We use it to introduce the basic principles of the model, key parameters and main results. Then we discuss the broader family of dynamical systems to which this model belongs.

Following our previous work $(80,123)$, responses of a population of $\mathrm{V} 1$ neurons are modeled as dynamical processes that evolve over time in a recurrent circuit (Fig. 2). The circuit is composed of different cell types. First and foremost is a population of principal cells (i.e., V1 simple-cells and complex-cells). The output firing rates these principal cells depend on the sum of two terms: 1) input gain (Fig. 2, orange) multiplied by input drive (Fig. 2, blue), and 2) recurrent gain (Fig. 2, purple) multiplied by recurrent drive (Fig. 2, green). The input drive is a weighted sum of the responses of population of input neurons, and the input gain is specified by a con-

Figure 2. Recurrent circuit. Orange, input gain. Blue, input drive. Purple, recurrent gain. Green, recurrent drive. Solid circles represent neurons. Different cell types: $x_{k}$, LGN inputs; $y_{j}$, principal cells; $u_{j}$ and $a_{j}$, modulator cells; small circles, inhibitory interneurons. Thin lines with arrow heads, axons. Solid lines, excitatory connections. Dashed lines, inhibitory connections. Circle heads, modulatory (e.g., shunting) connections. Thick fuzzy lines without arrow heads, dendritic compartments. Synaptic weights: $\mathbf{W}_{z x}$, orientation-selective weights determine input drive to simple-cells; $\mathbf{W}_{\hat{y} y}$, recurrent weights; W, normalization weights. Dendritic computations (sum of synaptic currents): $z_{j}$, input drive; $\hat{y}_{j}$, recurrent drive. Thick horizontal bar, input stimulus. Dotted circles superimposed on horizontal bar, LGN receptive field locations. Dashed circles, magnified view of LGN inputs with center-surround receptive fields.

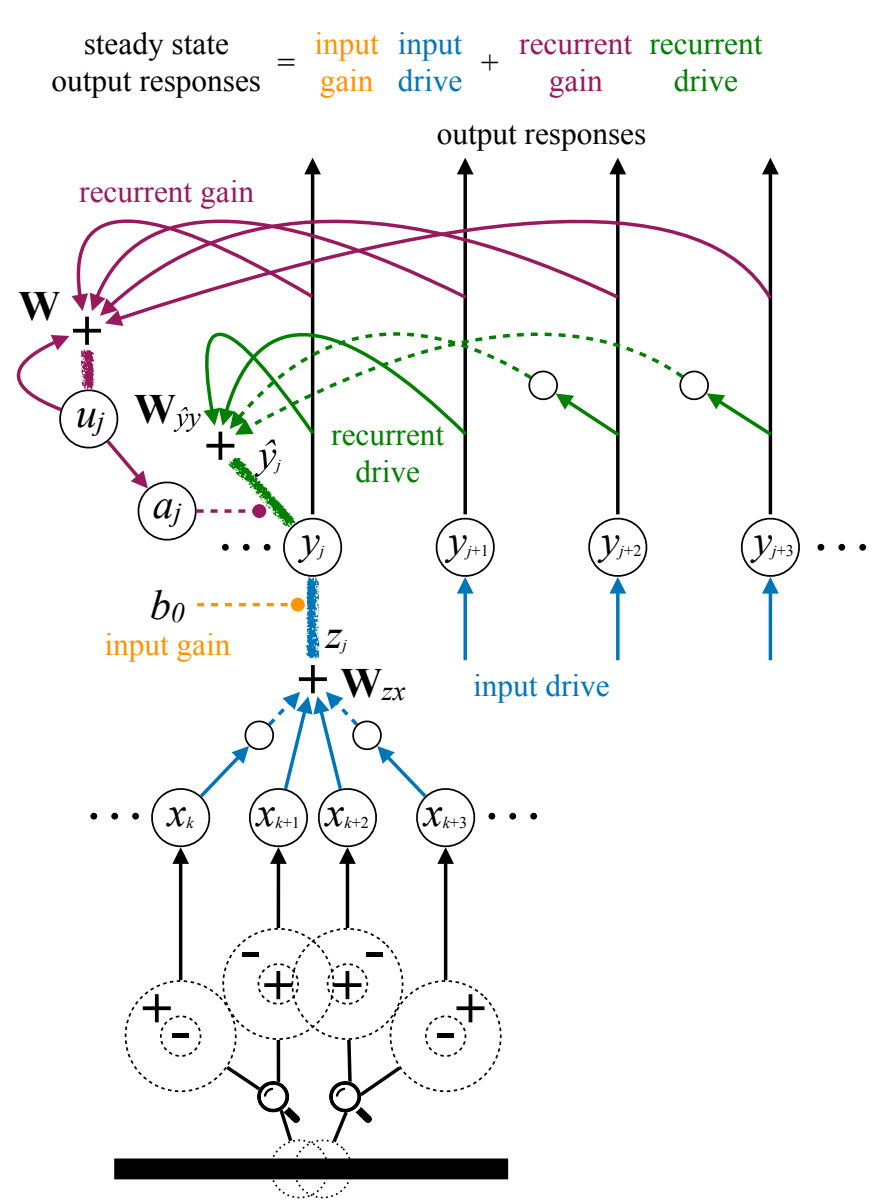


bioRxiv preprint doi: https://doi.org/10.1101/2020.03.22.002634; this version posted May 30, 2020. The copyright holder for this preprint (which was not certified by peer review) is the author/funder. All rights reserved. No reuse allowed without permission.

\section{Dynamic Normalization}

May 30, 2020

stant. These input neurons are presumed to be in the lateral geniculate nucleus (LGN) of the thalamus which, in turn, receive their inputs from neurons in the retina that respond with center-surround receptive fields to a visual stimulation (Fig. 2, thick horizontal bar). The recurrent drive is a weighted sum of principal cell responses, and the recurrent gain depends on the responses of a population of modulator cells. (We use the term "modulator" to mean a multiplicative computation regardless of whether or not it is implemented with neuromodulators). The responses of the modulator cells also depend on the principal cell responses (Fig. 2, purple).

A key feature of the model is that there are two nested recurrent loops that oppose each other. 1) Recurrent drive. The recurrent drive is a weighted sum of the principal cell responses, and the principal cells responses depend on the recurrent drive (Fig. 2, green). 2) Recurrent gain. The recurrent gain depends inversely on the modulator cell responses, and the modulator cell responses depend on a sum of principal cell responses (Fig. 2, purple). The recurrent drive is multiplied by the recurrent gain so that the modulators control the amount of recurrent amplification. Increasing the principal cell responses causes the modulator cells to increase their responses which causes the amount of recurrent amplification to decrease. Therefore, as the activity of the principle cells increases, the first recurrent loop increases the amount of recurrent amplification while the second loop decreases the amount of recurrent amplification. These two recurrent loops oppose each other such that the activity of the circuit may achieve a fixed point at which the neural activity is normalized. The responses at this fixed point typically exhibit one of two kinds of dynamics. If the modulator cells are sluggish then the responses of the principal cells can exhibit an initial transient overshoot before achieving the fixed point. If instead the modulator cells have a short time constant and a delay, then the fixed point may be unstable and the responses may exhibit oscillations.

\begin{tabular}{|c|c|c|}
\hline Symbol & Description & Possible mechanism \\
\hline $\mathbf{x}=\left(x_{1}, x_{2}, \ldots, x_{i}, \ldots, x_{M}\right)$ & Inputs & Firing rates of LGN cells \\
\hline $\mathbf{y}=\left(y_{1}, y_{2}, \ldots, y_{j}, \ldots, y_{N}\right)$ & Principal cell responses & Firing rates of pyramidal cells \\
\hline $\mathbf{v}=\left(v_{1}, v_{2}, \ldots, v_{j}, \ldots, v_{N}\right)$ & $\begin{array}{l}\text { Principal cell membrane potential } \\
\text { (deviation from rest in the absence of } \\
\text { stimulation) }\end{array}$ & $\begin{array}{l}\text { Input drive and recurrent drive computed in separate } \\
\text { compartments of dendritic tree }\end{array}$ \\
\hline $\mathbf{z}=\left(z_{1}, z_{2}, \ldots, z_{j}, \ldots, z_{N}\right)$ & Input drive & Dendritic computation, sum of synaptic currents \\
\hline$\hat{\mathbf{y}}=\left(\hat{y}_{1}, \hat{y}_{2}, \ldots, \hat{y}_{j}, \ldots, \hat{y}_{N}\right)$ & Recurrent drive & Dendritic computation, sum of synaptic currents \\
\hline $\mathbf{W}_{z x}$ & $\begin{array}{l}\text { Input weight matrix }(N \mathrm{x} M) \text { : each row } \\
\text { corresponds to the spatial RF of one } \\
\text { principal cell }\end{array}$ & $\begin{array}{l}\text { Excitatory and inhibitory (i.e., positive and negative) } \\
\text { synaptic weights }\end{array}$ \\
\hline $\mathbf{W}_{\hat{y} y}$ & $\begin{array}{l}\text { Recurrent weight matrix: each row } \\
\text { determines the recurrent drive for one } \\
\text { principal cell }\end{array}$ & $\begin{array}{l}\text { Excitatory and inhibitory (i.e., positive and negative) } \\
\text { synaptic weights }\end{array}$ \\
\hline $\mathbf{a}=\left(a_{1}, a_{2}, \ldots, a_{j}, \ldots, a_{N}\right)$ & $\begin{array}{l}\text { Modulator cell responses and recur- } \\
\text { rent gain }\end{array}$ & $\begin{array}{l}\text { Firing rates of inhibitory neurons (proportional to } \\
\text { membrane depolarization), each of which determines } \\
\text { conductance of the dendritic compartment of a princi- } \\
\text { pal cell receiving the recurrent drive }\end{array}$ \\
\hline $\mathbf{u}=\left(u_{1}, u_{2}, \ldots, u_{j}, \ldots, u_{N}\right)$ & $\begin{array}{l}\text { Responses of } 2 \text { nd population of mod- } \\
\text { ulator cell }\end{array}$ & $\begin{array}{l}\text { Firing rates of a type of excitatory neurons (propor- } \\
\text { tional to membrane depolarization, above a sponta- } \\
\text { neous firing rate) }\end{array}$ \\
\hline $\mathbf{W}$ and $w_{j k} \geq 0$ & $\begin{array}{l}\text { Normalization weight matrix } \mathbf{W} \text { com- } \\
\text { prising normalization weights } w_{j k}\end{array}$ & Excitatory synaptic weights \\
\hline$\tau_{v}, \tau_{a}, \tau_{u}$ & $\begin{array}{l}\text { Intrinsic time constants of each of the } \\
\text { corresponding cell classes }\end{array}$ & Membrane capacitance and conductance \\
\hline$b_{0}>0$ & Input gain (constant) & $\begin{array}{l}\text { Conductance of the dendritic compartment of the } \\
\text { principal cells receiving the input drive }\end{array}$ \\
\hline$\sigma>0$ & Contrast gain (constant) & Spontaneous firing rates of $\mathbf{u}$ modulator cells \\
\hline \multicolumn{3}{|c|}{$\begin{array}{l}\text { Table 1. Mathematical notation. Boldface lowercase letters denote vectors and boldface uppercase letters } \\
\text { denote matrices. The variables }(\mathbf{y}, \mathbf{v}, \hat{\mathbf{y}}, \mathbf{x}, \mathbf{z}, \mathbf{a}, \mathbf{u}) \text { are each functions time, e.g., } \mathbf{y}(t) \text {, but we drop the explicit de- } \\
\text { pendence on } t \text { to simplify the notation. }\end{array}$} \\
\hline
\end{tabular}


bioRxiv preprint doi: https://doi.org/10.1101/2020.03.22.002634; this version posted May 30, 2020. The copyright holder for this preprint (which was not certified by peer review) is the author/funder. All rights reserved. No reuse allowed without permission.

Dynamic Normalization

May 30, 2020

Recurrent normalization, as depicted in Fig. 2, agrees with experimental results suggesting that normalization operates via recurrent amplification (103-106). The recurrent drive involves both excitation and inhibition (Fig. 2, green solid and dashed lines, respectively). The modulator cells control the amount of recurrent amplification (Fig. 2, purple line with circle head). Consequently, both excitatory and inhibitory recurrent signals are amplified by an amount that is controlled by the modulator cells (Fig. 2, purple).

The remainder of this subsection walks through the equations of the dynamical system corresponding to the circuit model in Fig. 2 (see SI Appendix for additional details). In the subsections that follow, we demonstrate that this model mimics experimental observations of the dynamics of neural activity. We present the model as a computational theory for the computations performed by neural circuits in $\mathrm{V} 1$, not how they are implemented (but see Table 1 and Discussion for possible mechanisms).

Specifically, the responses of the principal cells are modeled by the following dynamical system (see SI Appendix of Ref. (80) for a primer on recurrent neural integrators):

$$
\begin{aligned}
& \tau_{v} \frac{d v_{j}}{d t}=-v_{j}+\left(\frac{b_{0}}{1+b_{0}}\right) z_{j}+\left(\frac{1}{1+a_{j}}\right) \hat{y}_{j}, \\
& y_{j}=\left\lfloor v_{j}\right\rfloor^{2}, \\
& \mathbf{z}=\mathbf{W}_{z x} \mathbf{x} \\
& \hat{\mathbf{y}}=\mathbf{W}_{\hat{y} y} \sqrt{\mathbf{y}} .
\end{aligned}
$$

See Table 1 for a description of the mathematical symbols and possible biological mechanisms. Vector $\mathbf{y}=\left(y_{1}, y_{2}\right.$, $\left.\ldots, y_{j}, \ldots, y_{N}\right)$ represents the firing rate responses of the principal cells, where the subscript $j$ indexes different neurons in the population, with different RF centers, orientation preferences, and spatial and temporal phases. The underlying membrane potentials of the principal cells are represented by vector $\mathbf{v}$. Membrane potential of the $j^{\text {th }}$ principal cell $v_{j}$ depends on a sum of two terms (Eq. 1): 1) input gain multiplied by input drive $z_{j}$ and 2) recurrent gain multiplied by recurrent drive $\hat{y}_{j}$. The input drive $z_{j}$ is a weighted sum of LGN inputs (Eq. 3; Fig. 2, blue; see $S I$ Appendix for details). The rows of the weight matrix $\mathbf{W}_{z x}$ determine the spatial RFs of the simple-cells

(Figs. S1b,c,d; see SI Appendix for details). The recurrent drive $\hat{y}_{j}$ is a weighted sum (with recurrent weights $\mathbf{W}_{\hat{y} y}$ ) of the square-root of the responses of the principal cells $y_{j}$ (Eq. 4; Fig. 2, green; see SI Appendix for details). The input drive and the recurrent drive are each multiplied by a gain factor. The input gain is specified by a constant $b_{0}$. The recurrent gain depends on the responses of the modulator cells $a_{j}$, as detailed below. Half-squaring (halfwave rectification and squaring) in Eq. 2 is an expansive nonlinearity that approximates the transformation from the membrane potential of the principal cells to their firing rates. The square root in Eq. $\mathbf{4}$ is a compressive nonlinearity that approximates a transformation from firing rates to synaptic currents. tems:

The modulator cells, which control the amount of recurrent amplification, are also modeled by dynamical sys-

$$
\begin{gathered}
\tau_{a} \frac{d a_{j}}{d t}=-a_{j}+\sqrt{u_{j}}+a_{j} \sqrt{u_{j}}, \\
\tau_{u} \frac{d u_{j}}{d t}=-u_{j}+\sum_{k} w_{j k} y_{k} u_{k}+\left(\frac{\sigma b_{0}}{1+b_{0}}\right)^{2},
\end{gathered}
$$

noting that all of the variables in these equations are constrained to be $\geq 0$. Vectors a and $\mathbf{u}$ represent responses of the two types of modulator cells (firing rates proportional to membrane depolarization, i.e., without squaring unlike Eq. 2). The need for both classes of modulator cells is explained below (see Variants of the model). Modulator cell responses $u_{j}$ represent a normalization pool, computed from the normalization weights $w_{j k}$ and the principal cell responses $y_{j}$ (Eq. 6; Fig. 2, purple). Responses of the other population of modulator cells $a_{j}$ are multiplied by the recurrent drive $\hat{y}_{j}$ (Eq. 1), thereby determining the recurrent gain and recurrent amplification. Responses $a_{j}$ depend on responses $u_{j}$ (Eq. 5), so that the recurrent amplification depends on the normalization pool. ized:

When the input drive is constant over time, the model has a fixed point such that the neural activity is normal-

$$
\mathbf{y}=\frac{\lfloor\mathbf{z}\rfloor^{2}}{\sigma^{2}+\mathbf{W} \mathbf{z}^{2}},
$$




\begin{abstract}
Figure 3. Recurrent amplification, effective time constant, onset transients, and oscillations. a. Response amplitudes of simulated simple- and complexcells as a function of grating contrast. Different colors correspond to different orientation preferences. b. Response amplitudes of the modulator cells: $a$ (dark gray curve) and square root of $u$ (light gray curve). c. Effective gain (i.e., the ratio of $y$ to $z^{2}$ ). d. Effective time constant. e. Response dynamics of simulated complex-cells for a sequence of stimulus contrasts. Thick horizontal bars, each stimulus presentation was 250 ms. Stimulus contrasts: 5, 10, 20, 50, and $100 \%$. Different colors correspond to different orientation preferences. Open circles, steady state response amplitudes (from panel a). Modulator cell time constant $\tau_{u}=10 \mathrm{~ms}$. f. Response dynamics of simulated complex-cells for $\tau_{u}=1 \mathrm{~ms}$. g. Membrane potential responses $(v)$ of simulated simple-cells for $\tau_{u}=1 \mathrm{~ms}$. Different colors correspond to different orientation preferences, all with the same temporal phase. $\mathbf{h}$. Membrane potential responses of simulated simple-cells, but with recurrent amplification disabled (note smaller y-scale).
\end{abstract}

where the numerator is half-squared, and the quotient means element-by-element division. Indeed, the exact form of Eqs. 1-6 was designed so that it would achieve this fixed point. To derive Eq. 7, set the derivatives in Eqs. 1, 5, and 6 equal to 0 and simplify (see SI Appendix). The values of $w_{j k}$ in Eq. 6 are the normalization weights, i.e., the elements of $\mathbf{W}$ in Eq. 7. Variants of Eq. 7 (with various exponents) have been fit to a wide range of experimental data (see SI Appendix for references).

Simulated neural responses in the following figures are intended to exhibit qualitative aspects of neurophysiological phenomena, i.e., the models have not (yet) been optimized to replicate published data by tuning or fitting the model parameters (see SI Appendix). We simulated responses to drifting sinusoidal gratings (or pairs of gratings) with various orientations, temporal frequencies, and contrasts. Responses to transient drifting gratings are more sustained than the responses to transient stationary gratings $(124,125)$. Unless otherwise stated, model parameters were: $b_{0}=0.2, \sigma=0.1, \tau_{v}=1 \mathrm{~ms}, \tau_{a}=2 \mathrm{~ms}, \tau_{u}=1 \mathrm{~ms}$. The normalization pool included all orientations (evenly weighted) at the center of a neuron's RF, and included only orientations near the preferred orientation at spatial locations surrounding the RF. Euler's forward method was used to compute Eqs. 1, 5, and $\mathbf{6}$ with time step $\Delta t=0.1 \mathrm{msec}$.

\section{Recurrent amplification, effective time constant, onset transients, and oscillations}

The recurrent circuit model (expressed by Eqs. 1-6 and depicted in Fig. 2) mimics many features of the dynamics of $\mathrm{V} 1$ activity. We focus on response dynamics because the mean firing rates are given by Eqs. 7-8 which are already known to fit a wide range of experimental data (Fig. 1) (see SI Appendix for references).

Simulated responses to grating stimuli with various contrasts replicated experimental observations (Fig. 3). Response amplitudes of simulated simple- and complex-cells were exactly equal to Eq. 8, saturating at high contrasts (Figs. 3a,e,f). The responses of the modulator cells increased monotonically with contrast but did not saturate (Fig. 3b). Responses were amplified by 100x when contrast was low but by only $\sim 1 \mathrm{x}$ when contrast was high (Fig. 3c), following Eq. 9. The effective time constant was correspondingly long for low contrasts but short for high contrasts (Fig. 3d), following Eq. 10. Consequently, high-contrast stimuli evoked rapid increases in activity, whereas low-contrast stimuli evoked much slower and more gradual increases in activity before achieving steady state (Fig. 3e). The rate at which activity decreased following stimulus offset was different from the rate at which activity increased after lifting off from zero following stimulus onset (Fig. 3e). These results are similar to a variety of electrophysiological measurements $(52,55,63,100,124-129)$. 
bioRxiv preprint doi: https://doi.org/10.1101/2020.03.22.002634; this version posted May 30, 2020. The copyright holder for this preprint (which was not certified by peer review) is the author/funder. All rights reserved. No reuse allowed without permission.

Dynamic Normalization

May 30, 2020

We can derive expressions for the effective gain and the effective time constant of the responses, to generate experimentally-testable predictions and for fitting data. The effective gain and effective time constant both decrease with increasing stimulus strength. Weak stimuli are strongly amplified (large effective gain) via the recurrent circuit which takes a period of time (long effective time constant). Strong stimuli are weakly amplified (small effective gain) which happens more quickly (short effective time constant). The effective gain of each neuron in the circuit (the ratio of each element of $\mathbf{y}$ to each element of $\mathbf{z}^{2}$ ) depends on the input drive and the normalization weights: $\mathbf{W z}^{2}$. The effective time constant depends on the effective gain (see SI Appendix) so it too depends on $\mathbf{W z}^{2}$. For stimuli comprised of drifting sinusoidal gratings or pairs of gratings:

$$
\begin{aligned}
& r \propto \frac{c_{t}^{2}}{\sigma^{2}+c_{t}^{2}+\beta c_{m}^{2}}, \\
& g=\frac{1}{\sigma^{2}+c_{t}^{2}+\beta c_{m}^{2}}, \\
& \tau=\tau_{v}\left(\frac{1+b_{0}}{b_{0}}\right) \sqrt{g},
\end{aligned}
$$

where $r$ is the amplitude of a principal cell's response (e.g., the mean firing rate of a V1 complex-cell), $g$ is the effective gain of that neuron's responses, and $\tau$ is that neuron's effective time constant (see SI Appendix for derivations). The value of $c_{t}$ is the contrast of a test grating (e.g., a preferred orientation grating restricted to the RF). The value of $c_{m}$ is the contrast of a mask grating that by itself does not evoke a response. The value of $0<\beta<1$ depends on the normalization weights. Eqs. 8-10 follow from Eq. 7 because the input drive is weighted sum of the input, i.e., $z_{j}$ is proportional to contrast. From Eq. 8, it is evident that responses saturate (level off) when the test contrast is large $(\gg \sigma)$, cross-orientation suppression results when a mask grating is superimposed that is orthogonal to the preferred orientation, and surround suppression results when a mask grating is added in the region surrounding the RF, all characteristics of visual neurophysiology (Fig. 1). From Eqs. 9-10, it is evident that these stimulus components also have an impact on the timing of the responses.

By changing one of the model parameters (specifically, the intrinsic time constant of the modulator cells $u_{j}$ ), simulated responses to high contrast stimuli exhibited either strong transients following stimulus onset (Fig. 3e, $\left.\tau_{u}=10 \mathrm{~ms}\right)$ or stable, high-frequency $(40-50 \mathrm{~Hz})$ oscillations (Fig. 3f, $\left.\tau_{u}=1 \mathrm{~ms}\right)$. Both of these phenomena - onset transients $(124,125,130)$ and stable oscillations $(35,87,89-102)$ - have been widely reported from experimental observations.

For some parameter regimes, the responses exhibit onset transients (Fig. 3e) followed by stable oscillations (Fig. 3f), but we have not systematically characterized the parameters that do so. The temporal filter that is used to simulate the responses of the LGN inputs (see SI Appendix) attenuates the onset transients. Without that temporal filter, there would typically be an initial transient overshoot.

In these simulations, the normalization weights were all equal, so the response transients and/or oscillations were synchronized across the population of neurons. Consequently, in spite of the complex dynamics, ratios of the responses across neurons with different orientation preferences were maintained throughout each stimulus presentation, resembling some experimental results (125), and enabling an accurate readout of the stimulus orientation at any time point. With unequal (non-negative) normalization weights, response ratios evolved over time with non-stationary readout, analogous to other experimental results (131). Furthermore, with unequal normalization weights, response ratios also depended on stimulus contrast so that the simulated neural responses did not exhibit perfectly contrast-invariant tuning curves.

Disabling the recurrent amplification (i.e., simulating an experiment in which cortical spiking is shut down) attenuated the membrane potential response amplitudes by a factor of $\sim 10 \mathrm{x}$ at high contrasts (Fig. 3g,h), while maintaining their orientation-selectivity, resembling electrophysiological results (132-135).

\section{Temporal-frequency tuning and phase advance depend on contrast}

Temporal-frequency tuning of both simple- and complex-cells depends on stimulus contrast, and simple-cell response phase depends on contrast $(6,55,84-86)$. It was previously proposed that these phenomena can be explained by a recurrent normalization model in which a neuron's conductance (and consequently its intrinsic time constant) depends on stimulus contrast $(6,55)$. Here, we hypothesize instead that the effective time constant depends on contrast because the amount of recurrent amplification in the circuit decreases with increasing contrast (Eqs. 9-10).

Simulated temporal-frequency tuning depended systematically on contrast, responding to a broader range of temporal frequencies at high contrasts (Fig. 4). Figs. 4a,b plot results for a population of neurons with preferred 

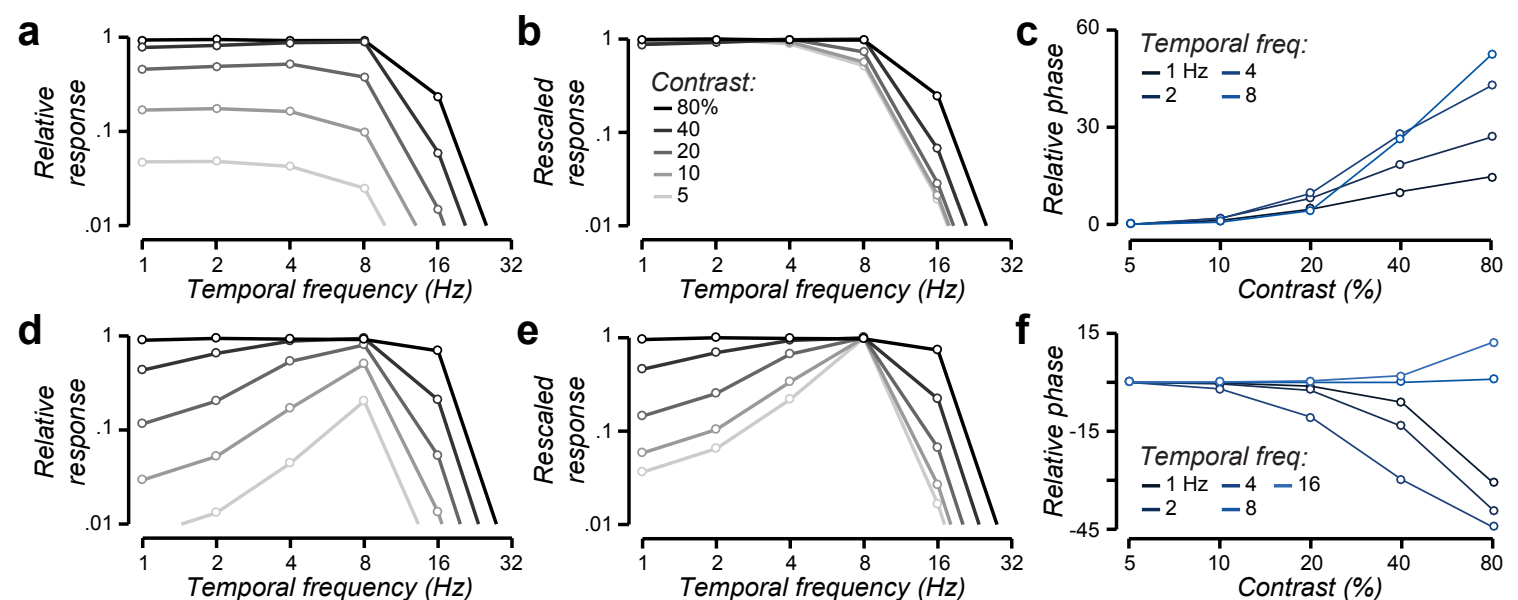

Figure 4. Temporal-frequency tuning and phase advance depend on contrast. a-c. Lowpass temporal-frequency tuning $(\omega=0 \mathrm{~Hz})$. a. Response amplitudes for each of several stimulus temporal frequencies. Different shades of gray correspond to different stimulus contrasts: $5,10,20,40,80 \%$. b. Rescaled responses. The different curves (from panel a) are each rescaled to have the same maximum so that the shapes of the curves can be readily compared. Responses to high contrasts (darker curves) are elevated compared to the responses to low contrasts (lighter curves). c. Phase advance. Response phase for each of several contrasts. Different colors correspond to a different temporal frequencies: $12,4,8 \mathrm{~Hz}$. d-f. Bandpass temporal-frequency tuning $(\omega=8 \mathrm{~Hz})$.

temporal frequency $\omega=0 \mathrm{~Hz}$, i.e., the recurrent drive in the model acted like a lowpass filter (see SI Appendix). Increasing stimulus contrast increased the responsivity of the simulated neurons for high temporal frequencies.

Figs. 4d,e plot results for neurons with preferred temporal frequency $\omega=8 \mathrm{~Hz}$, i.e., the recurrent drive in the model acted like a bandpass filter, matching the preferred temporal frequency of the simulated LGN inputs. In this case, increasing stimulus contrast increased the responsivity of the simulated neurons for both low and high temporal frequencies. For low contrasts, temporal-frequency tuning was bandpass with a relatively narrow bandwidth. Increasing stimulus contrast transformed the temporal frequency tuning from bandpass to lowpass while nearly doubling the high temporal-frequency cutoff. This behavior arises in the model because the effective time constant depends on contrast: the effective gain decreases with increasing contrast (Eq. 9) and the effective time constant decreases with decreasing effective gain (Eq. 10). A shorter time constant corresponds to a broader bandwidth, raising the high temporal-frequency cutoff for a lowpass tuning curve, and raising both the low and high cutoffs for a bandpass tuning curve.

Response phase also depended systematically on contrast (Figs. 4c,f). For simulated simple-cells with lowpass temporal-frequency tuning, response phases advanced with increasing contrast, more so for higher temporal frequencies (Fig. 4c). For simulations with bandpass temporal-frequency tuning, response phases shifted in opposite directions for temporal frequencies above and below the preferred temporal frequency (Fig. $\mathbf{4 f}$ ).

Results like those shown in Figs. 4a-c have been observed experimentally (6, 55, 84-86): increasing phase advance and increasing the high temporal-frequency cutoff with increasing contrast. The model predicts that the effects shown in Figs. 4d-f may be evident for neurons with narrow temporal-frequency tuning, e.g., perhaps direction-selective neurons in layer $4 \mathrm{~b}$.

\section{Response dynamics depend on stimulus location}

The dynamics of V1 activity depends on whether a stimulus is placed in the center or flanks of a neuron's receptive field (83). Activity evoked by a small grating patch extends over a cortical region of several millimeters (depending on stimulus size, spatial frequency, and eccentricity). Following stimulus onset, responses rise simultaneously over the entire active region, but reach their peak more rapidly at the center. Furthermore, the rate of response increase following stimulus onset is faster for higher contrasts. Following stimulus offset, responses fall simultaneously at all locations, and the rate of response decrease is the same for all locations and all contrasts. It was previously proposed that these phenomena can be explained by a recurrent normalization model in which a neuron's conductance (and consequently its intrinsic time constant) depends on the spatial distribution of stimulus contrasts, via the normalization weights (83). Here, we hypothesize instead that the effective time constant (as opposed to the intrinsic time constant) of each neuron depends on normalization weights.

Simulated responses recapitulated the experimentally-measured spatiotemporal dynamics (Fig. 5). Responses lifted off simultaneously following stimulus onset, but increased at a faster rate for RF locations centered on the stimulus (Fig. 5, darker colors), and for higher contrasts (Fig. 5, responses to $2^{\text {nd }}$ stimulus presentation at 


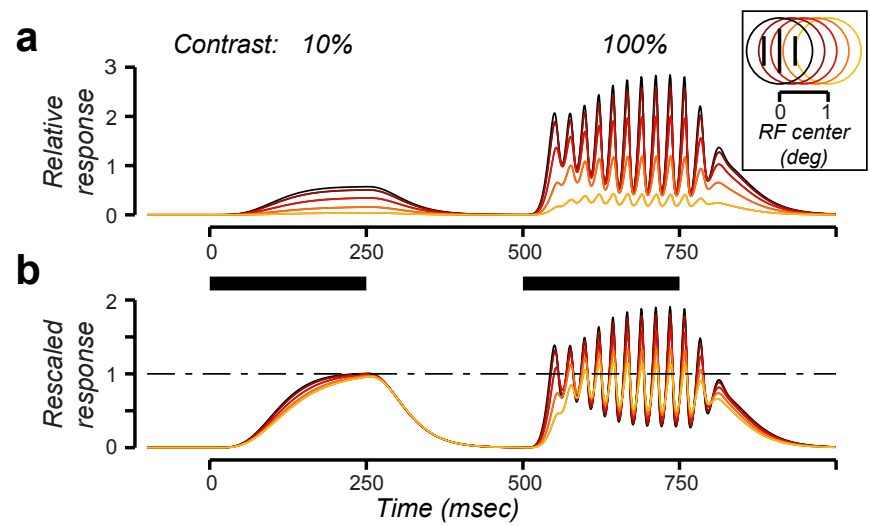

Figure 5. Response dynamics depend on stimulus location. a. Response dynamics of simulated complex-cells for two contrasts (10\% and $100 \%)$. Different colors correspond to different RF centers. Thick horizontal bars, stimulus presentations. Inset, spatial arrangement of stimulus and RF locations. $\mathbf{b}$.

Rescaled responses. The different curves (from panel a) are each rescaled by the corresponding fixed point, so that the shapes of the curves can be readily compared. The rate of response increase following stimulus onset is faster for stimuli placed in the center of a receptive field (darker colors) and slower on the flanks of the receptive field (lighter colors).

$t=500 \mathrm{~ms}$ ). Recurrent amplification was weaker when the stimulus was presented closer to the center of a neuron's RF, and it was weaker for higher contrasts. Consequently, the effective gain was smaller (Eq. 9) and the time constant was shorter (Eq. 10) for these conditions. The effective time constant following stimulus offset was $\sim 60 \mathrm{~ms}$, regardless of what the stimulus had been (Eqs. 9-10 with $c_{t}=c_{m}=0, b_{0}=0.2, \sigma=0.1$, and $\tau_{v}=1 \mathrm{~ms}$ ).

\section{Oscillations depend on stimulus contrast and size}

Simulated responses exhibited oscillations at high frequencies (Fig. 3f). For grating stimuli, and in the absence of noise, these oscillations were evident only at high (>50\%) contrasts, and the oscillation amplitudes (Fig. 6b) increased with stimulus size and contrast. Oscillation frequencies also increased with contrast. Response amplitudes, on the other hand, exhibited surround suppression so they were non-monotonic with stimulus size at high contrasts (Fig. 6a, dark gray and black curves). The oscillations depended indirectly on stimulus temporal frequency because the input drive to each neuron depended on stimulus temporal frequency with respect to the neurons' preferred temporal frequency (Fig. 4). That is, a lower contrast grating with a temporal frequency at the peak of the tuning curve generated the same oscillations as a higher contrast with a temporal frequency on the flank of the tuning curve, such that the two stimuli evoked the same input drive amplitudes. But the oscillations were otherwise (beyond the dependence on input drive amplitudes) independent of stimulus temporal frequency.

Oscillations were also evident for high contrast plaid stimuli, composed of a pair of orthogonal gratings, but the oscillations generated by plaids were smaller in amplitude and lower in frequency than those generated by gratings of the same contrast. Responses to plaids exhibited cross-orientation suppression; the response evoked by a $50 \%$ contrast grating with a neuron's preferred orientation was suppressed by about a factor of 2 when an orthogonal mask grating (also $50 \%$ contrast) was superimposed (Fig. 6a, dashed blue curve vs. $3^{\text {rd }}$ to darkest gray curve). Oscillation amplitudes generated by $100 \%$ contrast plaids were about midway between those generated by $50 \%$ and $100 \%$ contrast gratings (Figs. 6b, dashed blue curve).

The oscillations depended on the strength of the normalization pool: specifically, the product of the normalization weights and the input drive $\mathbf{W z}^{2}$. The normalization pool increased with contrast because the input drive $\mathbf{z}$ was proportional to contrast. The normalization pool increased with stimulus size because it comprised a weighted sum (with non-negative weights) over space.

Figure 6. Oscillations depend on stimulus contrast and size. a. Surround suppression and cross-orientation suppression. Gray curves, response amplitudes to preferred-orientation gratings with each of several stimulus sizes. Different shades of gray correspond to different stimulus contrasts: $6.25,12.5,25,50$, and $100 \%$. Dashed blue curve, $100 \%$ contrast plaid composed of a pair of orthogonal gratings (each $50 \%$ contrast). b. Oscillation amplitudes (computed by summing the Fourier amplitudes of the responses between $30-200 \mathrm{~Hz}$, ignoring the first $250 \mathrm{~ms}$ of the responses after stimulus onset). Oscillations are evident only for the highest contrasts and increase with both stimulus contrast and stimulus size.

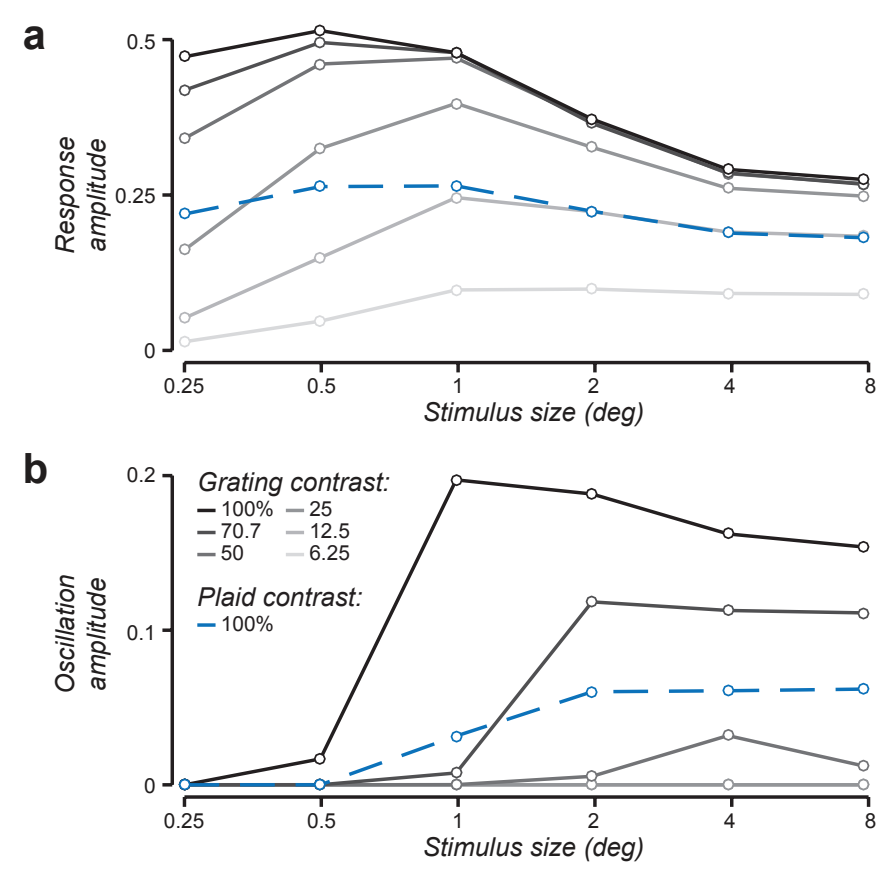


bioRxiv preprint doi: https://doi.org/10.1101/2020.03.22.002634; this version posted May 30, 2020. The copyright holder for this preprint (which was not certified by peer review) is the author/funder. All rights reserved. No reuse allowed without permission.

Dynamic Normalization

May 30, 2020

The normalization pool was smaller for a $100 \%$ contrast plaid than a $100 \%$ contrast grating. If the normalization was untuned such that all of the normalization weights were 1 , then the normalization pool for a $100 \%$ contrast plaid composed of two $50 \%$ contrast gratings $\left(\mathbf{W z}^{2}=0.5^{2}+0.5^{2}\right)$ would have been equal to that for a $70.7 \%$ contrast grating $\left(\mathbf{W z}^{2}=0.707^{2}\right)$. The simulated oscillations differed for these two stimulus conditions (Figs. $\mathbf{6 b}$, dashed blue curve vs. $2^{\text {nd }}$ to darkest gray curve) because the normalization pool included all orientations (evenly weighted) at the center of each neuron's RF, and only orientations near the preferred orientation at surrounding locations.

All of these results are commensurate with experimental observations that oscillation amplitudes and frequencies depend systematically on stimulus contrast, size, and spatial pattern (35, 87, 89-102), and that oscillations are linked to normalization $(35,87,88)$. Like the simulation results, oscillation amplitudes in $\mathrm{V} 1$ increase with stimulus contrast and size, oscillation frequencies increase with stimulus contrast, and oscillation amplitudes are smaller for plaids than for gratings (and even smaller for stimuli composed of multiple components, also predicted by the model).

Using the current model configuration, simulated oscillation frequencies increased with stimulus size, however, unlike the experimental measurements that decreased with stimulus size $(35,87,98)$. Previous models have tackled this problem by incorporating a mechanism that pools over large spatial regions that provides excitatory feedback to the principal cells $(98,136)$. The current family of models may, likewise, be extended by enhancing the recurrent drive with an additional weighted sum over a larger region of the visual field (80). We have verified that doing so may explain the observed decrease in oscillation frequency with increasing stimulus size.

\section{Phase space trajectories and bifurcation analysis}

Oscillations emerged for some parameter regimes of the model, not others, and oscillations in the gamma frequency band corresponded to restricted ranges of those parameter regimes. A bifurcation analysis was performed to determine ranges of parameter values for which oscillations occur and to determine the corresponding oscillation frequencies.

We analyzed a reduced version of the model in which each of the variables was a scalar instead of a vector (SI Appendix, Eq. S37), i.e., one neuron of each of the 3 types $(y, a$, and $u$ ) instead of a population of neurons with different RF centers and orientation preferences. We characterized the dynamics of the model as a function of the input drive $(z)$, the intrinsic time constants of the modulator cells $\left(\tau_{a}\right.$ and $\left.\tau_{u}\right)$, and the input gain $\left(b_{0}\right)$. In this reduced model, the input was a step at time $t=0$ and maintained a constant value thereafter.

The model exhibited distinct behaviors with boundaries (state transitions) between them (Fig. 7). When the input drive was small, the fixed point was stable (i.e., an attractor) and simulated responses $(y)$ achieved steady state with no oscillations (Fig. 7a, green point; Fig. 7b). When the input drive was large, the fixed point was unstable with a stable limit cycle and responses exhibited stable oscillations (Fig. 7a, red point and dotted gray curves; Fig. 7d). For a middle range of input drives, the fixed point was a spiral attractor and responses exhibited oscillations transiently before achieving steady state (Fig. 7a, yellow point; Fig. 7c). The steady state responses increased monotonically with input drive until the bifurcation, at which point the responses exhibited stable oscillations around the fixed point and no longer achieved a steady state (Fig. 7a, intersection of solid black, dashed black, and dotted gray curves).

The input drive that induced a bifurcation depended systematically on model parameters (Figs. 7e). Each panel of Fig. 7e depicts a 2D bifurcation diagram, i.e., a 2D slice through the space of model parameters. Each panel indicates the input drives for which bifurcations occurred (solid black curves) for different values of $\tau_{u}$, and the different panels correspond to different values of $\tau_{v}$ and $\tau_{a}$. Also indicated are the oscillation frequencies (gray scale) when the model exhibited stable oscillations or zero (white) otherwise.

\section{Variants of the model}

The dynamical system expressed by Eqs. 1-6 is but one example of a family of circuit models of normalization, each of which implements normalization via recurrent amplification (see SI Appendix for several examples of alternative models from this family). Some of these various models exhibit qualitatively different dynamics such that measurements of the dynamics of neural activity in V1 may be used to distinguish between the alternatives. Each of the various models in this family imply different circuits, such that they may be distinguished experimentally using cell-type specific indicators.

For example, one of these variants can be ruled out as a plausible model of $\mathrm{V} 1$ activity because it does not exhibit dynamics commensurate with V1 activity. This variant (see SI Appendix, Eq. S35) is a simpler circuit with only two types of neurons, a principal cell and a single type of modulator cell instead of two. The circuit has a stable fixed point such that the primary neurons achieve steady state responses given by the normalization equation (Eqs. 7-8). We have been able to prove mathematically, over a very broad range of parameter values, that the fixed point is stable over the full range of input drives. That is, there is no parameter regime in which the respons- 


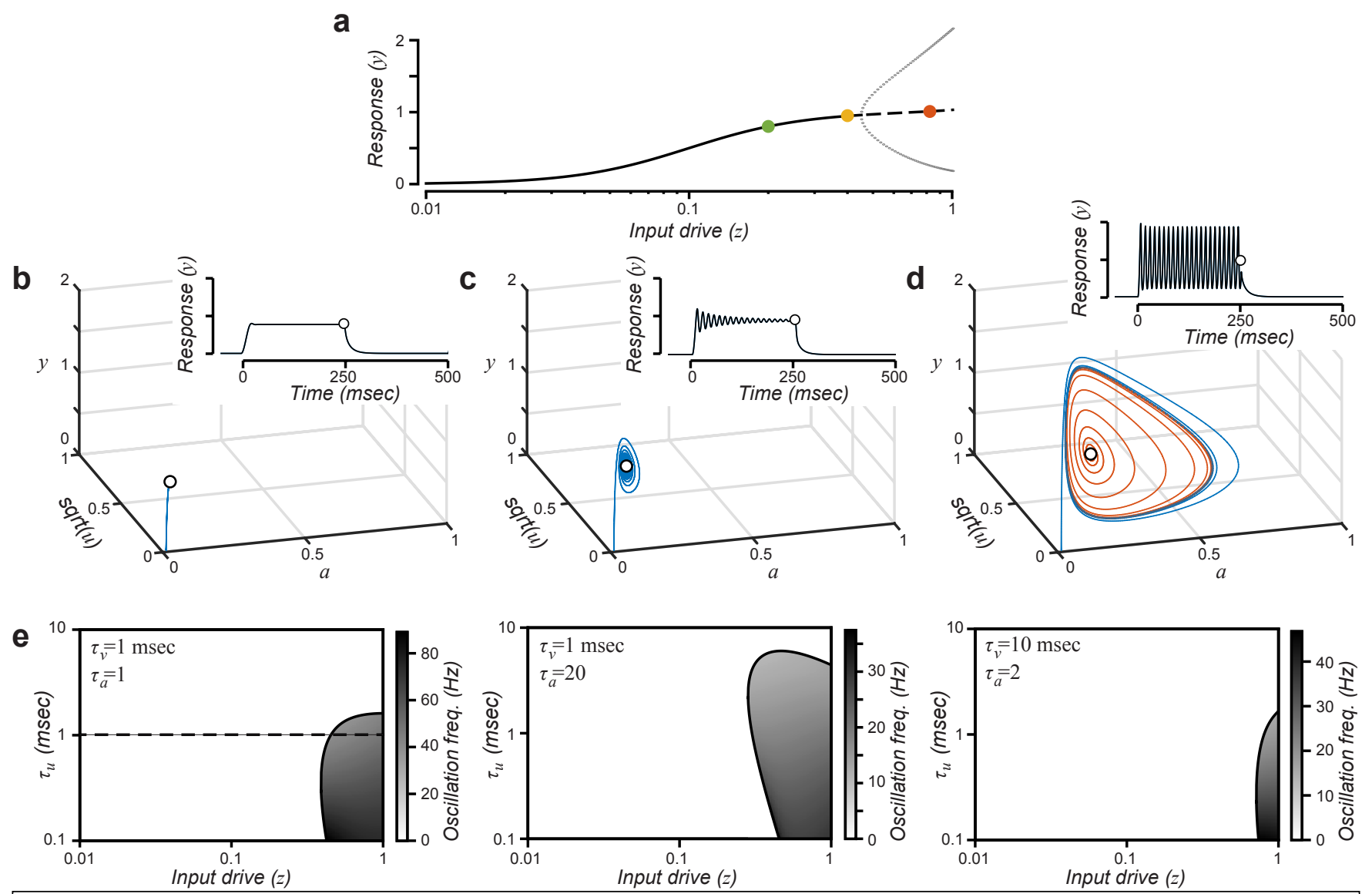

Figure 7. Phase space trajectories and bifurcation analysis. a. Bifurcation analysis as a function of input drive. Solid curve, stable fixed point (i.e., attractor). Dashed curve, unstable fixed point. Dotted gray curves, maximum and minimum values of $y$ during stable oscillations. Green, yellow, and orange dots correspond to phase space trajectories in panels $\mathbf{b}, \mathbf{c}$, and $\mathbf{d}$, respectively. b. Phase space trajectory convergent to a stable fixed point, corresponding to the green dot in panel a $(z=0.2)$. Blue curve, trajectory of responses of the 3 neurons $(y, a$, and $u$ ) starting from rest (zero). Open circle indicates the fixed point. Inset, response time course of principal cell $y$ (open circle, fixed point). c. Phase space trajectory convergent to a spiral attractor, corresponding to the yellow dot in panel a $(z=0.4)$. d. Phase space trajectories for an unstable fixed point with stable limit cycle, corresponding to the orange dot in panel a $(z=0.8)$. Blue curve, trajectory of responses of the 3 neurons starting from rest (zero). Orange curve, trajectory of responses of the 3 neurons for an initial condition that is a slight $(1 \%)$ perturbation from the fixed point. The blue curve spirals clockwise into the limit cycle and the orange curve spirals clockwise outward from near the fixed point to the limit cycle. The responses traverse a curved manifold (shaped like a saddle or potato chip). e. Bifurcation analysis as a function of input drive $(z)$ and time constant parameters $\left(\tau_{v}, \tau_{a}\right.$ and $\left.\tau_{u}\right)$. Other model parameters: $b_{0}=0.2, \sigma=0.1$. Responses in the shaded regions exhibit stable oscillations (unstable fixed points with stable limit cycles). Grayscale indicates oscillation frequency. Dashed line corresponds to panel a.

es exhibit stable oscillations (see SI Appendix).

An intuition for why two modulator cells are needed relies on the observation that the membrane equation acts as an exponential lowpass filter. A single exponential lowpass filter imposes a phase delay. A cascade of two lowpass filters in sequence also imposes a time delay (the peak of the impulse response function is delayed). This time delay suffices for oscillations to emerge (see SI Appendix, compare the system of Eq. S35 which does not oscillate with the system of Eq. S36 which does oscillate when the input drive is strong enough).

\section{Discussion}

We developed a family of circuit models of normalization. The key idea is that normalization operates via recurrent amplification, i.e., amplifying weak inputs more than strong inputs (103-106). The modulator cells determine the recurrent gain, thereby controlling the amount of recurrent amplification. Each of the models in this family exhibits output responses with a fixed point that follows the normalization equation (Eqs. 7-8) exactly, for arbitrary (non-negative) normalization weights. The normalization equation is already known to fit a wide range of ex- 
bioRxiv preprint doi: https://doi.org/10.1101/2020.03.22.002634; this version posted May 30, 2020. The copyright holder for this preprint (which was not certified by peer review) is the author/funder. All rights reserved. No reuse allowed without permission.

Dynamic Normalization

May 30, 2020

perimental data (see SI Appendix for references).

This family of models mimics experimental observations of $\mathrm{V} 1$ dynamics linked to normalization: onset transients, the contrast dependence of the rate of response increase following stimulus onset and response decrease following stimulus offset (Figs. 3 and $\mathbf{5}$ ), and the contrast dependence of temporal-frequency tuning and phase advance (Fig. 4). Furthermore, for some of the models in this family, the fixed point can become unstable for large, high-contrast narrow band stimuli like sinusoidal gratings. Under these circumstances, the circuit achieves a dynamic equilibrium (i.e., a limit cycle) and responses exhibit oscillations (see also Ref. 88). The oscillations emerge because of the recurrent circuitry, depending on the strength of the normalization pool (the product of the normalization weights and the corresponding input drive), thereby providing us with an explanation for why gamma oscillations are linked to normalization $(35,87,88)$. In spite of the complex dynamics, ratios of the simulated responses across neurons with different stimulus preferences may be maintained throughout each stimulus presentation, enabling an accurate readout of stimulus orientation (or other stimulus parameters) at any time point following the onset of the responses.

The recurrent circuit models presented here are examples of a class of circuit models called Oscillatory Recurrent Gated Neural Integrator Circuits (ORGaNICs) $(80,123)$. ORGaNICs are a generalization of and a biophysically-plausible implementation of Long Short Term Memory units (LSTMs), a class of artificial recurrent neural networks (137). LSTMs have been applied to a number of machine learning applications including video summarization, language modeling, translation, and speech recognition (e.g., 138, 139-141). By virtue of being a generalization of LSTMs, ORGaNICs inherit all of the capabilities LTSMs. Consequently, the theoretical framework proposed here actually works - it is capable of performing useful computations that solve real-world problems. ORGaNICs may also be used to explain key aspects of working memory and motor control. ORGaNICs may be used to explain the complex dynamics of delay-period activity during a working memory task, how information is manipulated (as well as maintained) during a delay period, and how that information is read out from the dynamically varying responses at any point in time in spite of the complex dynamics (80). When applied to motor systems, these circuits convert spatial patterns of premotor activity to temporal profiles of motor control activity: different spatial patterns of premotor activity evoke different motor control dynamics (80). ORGaNICs are also capable of prediction over time (123). The modulators in ORGaNICs perform multiple functions including normalization, controlling maintenance of a representation over time, controlling pattern generators, gated integration/updating, time warping, reset, controlling the effective time constant, controlling the relative contributions of bottom-up versus top-down connections, and weighting the reliability of sensory evidence (likelihood) and internal model (prior, expectation) for inference and multisensory integration $(80,123,142)$.

Here, we demonstrated that this same family of circuit models can simulate the dynamics of neural activity in V1. Consequently, this theoretical framework is applicable to diverse cognitive processes and neural systems, and we can use V1 as a model system for understanding the neural computations and circuits in many brain areas.

\section{Gamma oscillations}

Narrow-band gamma oscillations have been proposed to play a functional role in stimulus feature binding (143-146), attention (147-150), and/or synchronizing neuronal activity to enhance signal transmission and communication between brain areas $(146,149,151-158)$. These speculations have been met with considerable skepticism $(90,93,95,98,99,159-163)$, in part because oscillation amplitude depends strongly on stimulus conditions $(35,87,89-102)$, incommensurate with the perception of those stimulus conditions.

Gamma oscillations in the current family of models emerge from the nonlinear dynamics of the recurrent circuit. Synchronized spiking was not required to generate gamma oscillations. Gamma oscillations were generated for a restricted subset of stimulus conditions, depending on the strength of the normalization pool. Consequently, oscillation amplitude was strongest for large, high contrast gratings, and weaker (or non-existent) for other spatial patterns and low contrasts, similar to experimental results $(35,87,89-102)$.

Long wavelength stimuli have been found to generate particularly large amplitude gamma oscillations (101, 164). It should be straightforward to extend the current family of models to account for these results by including red-green and blue-yellow color-opponent channels (165-167) in the LGN input, and by setting the normalization weights to be large for the red-green channel.

The current theoretical framework is most similar to bifurcation-based models of gamma oscillations (168, 169), as opposed to the so-called pyramidal-interneuron gamma (PING) and interneuron gamma (ING) mechanisms for producing gamma oscillations (136, 162, 170-180). ING models generate oscillations with an interconnected network of inhibitory neurons (although some of these models rely on weak excitatory interconnections to synchronize the oscillations across multiple subpopulations of inhibitory neurons). In PING models, a volley of activity in the excitatory cells recruits a slightly delayed volley of activity in the inhibitory cells, which inhibits the excitatory cells for a gamma cycle, after which they recover. In both PING and ING models, oscillations are generated by neural circuits that behave as intrinsic oscillators. In bifurcation-based models (including ours), unlike 
bioRxiv preprint doi: https://doi.org/10.1101/2020.03.22.002634; this version posted May 30, 2020. The copyright holder for this preprint (which was not certified by peer review) is the author/funder. All rights reserved. No reuse allowed without permission.

Dynamic Normalization

May 30, 2020

PING and ING models, oscillations emerge as drive to the excitatory population increases so that a steady state loses stability via Hopf bifurcation. The appearance of oscillations critically depends on the relative timescales of excitation and inhibition. In both of the previous bifurcation-based models $(168,169)$, oscillation frequencies decrease with slower inhibition. In our models, decreasing the time constants of the modulatory cells likewise results in slower oscillations, but only up to a point. If the modulator cell time constants are either too slow or too fast, then bifurcations and oscillations are eliminated altogether (Fig. 7e). Analogous to PING models, we observed that the simulated oscillatory activity of modulator cells lagged $\left(\sim 90^{\circ}\right.$ phase) behind the activity of principal cells. Unlike any of previous models of gamma oscillations, we designed the current family of models to perform a function (normalization), and gamma oscillations emerged as a by-product.

\section{Failures and extensions}

Stable oscillations were observed in the simulation results for input drives (i.e., contrasts) above a threshold level (above the bifurcation), but narrow-band gamma power has been observed experimentally to change gradually with continuous parametric variation in stimulus parameters $(87,90,97,98)$. Weaker inputs evoked transient oscillations (spiral attractor dynamics, Fig. 7) after stimulus onset in our model simulations, and such transient oscillations could be confounded with stable oscillations in some experimental results (e.g., such transient oscillations may follow saccades). Furthermore, all the simulation results reported above were performed in the absence of noise. With noise added to the input drive, we observed stochastic resonance in the gamma frequency range, even for weak inputs below the bifurcation (Fig. S2, see SI Appendix). This suggests that gamma-band activity may be induced by broadband noise in neural activity $(136,162)$, because the noise spectrum is shaped by recurrent normalization to exhibit a resonant peak in the gamma-frequency range.

The effective time constants of the principal cells in our simulations ranged from $6-60 \mathrm{~ms}$, which is within a reasonable range for in vivo cortical neurons, but the values of the intrinsic time constants (1-2 ms) were extremely short. Increasing the values of the time constant parameters would make the responses sluggish. For example, setting $\tau_{v}=10 \mathrm{~ms}$ (while holding the other parameters unchanged) would mean that the effective time constant (the integration time) for low contrast stimuli would be as long as $600 \mathrm{~ms}$, which is unrealistic. Increasing the time constants would also decrease the oscillation frequencies (Fig. 7). For example, setting $\tau_{v}=10 \mathrm{~ms}$ (while holding the other parameters unchanged) would generate $\sim 15 \mathrm{~Hz}$ oscillations. This is a challenge for any model that relies heavily on recurrent amplification because the recurrence takes time (multiples of the time constant). It may help for some of the normalization to be feedforward and/or precortical (see Mechanisms), so that the cortical circuit need not responsible for all of the amplification. Furthermore, increasing the intrinsic time constant could be compensated for by increasing the value of the $b_{0}$ parameter so as to leave the effective time constant unchanged (Eq. 10). Doing so, however, would partially undermine the result illustrated in Fig. $\mathbf{3 h}$, that membrane potential response amplitudes are reduced by disabling the recurrent amplification; this result would still be evident at low contrasts (because of the half-squaring nonlinearity) but not at high contrasts.

Attention is associated with both increases in the gain of visually-evoked responses (e.g., 18, 21, 23) and increases in gamma oscillations $(35,147-150,155,160,181-185)$. The constant input gain parameter $b_{0}$ may be replaced by a variable vector $\mathbf{b}$ in Eq. 1 (while keeping the constant $b_{0}$ in Eq. 6), in combination with normalization, to model the effects of attention on sensory responses. The elements of $\mathbf{b}$ determine the relative attentional gain for each neuron in the circuit (i.e., with different RF centers and different orientation preferences). Extending the model in this way would yield steady state output responses that are already known to fit experimental measurements of response gain changes with attention (e.g., 23) (see SI Appendix for additional references). This change to the model would also affect the dynamics of the responses and may be used to explain the ostensible link between attention and gamma oscillations $(35,160)$.

Cross-orientation suppression is faster than surround suppression $(63,67)$. The current model may be extended to explain these results by incorporating an additional delay for contributions to the normalization pool from surrounding spatial locations. We hypothesize that a single computational process can explain both forms of suppression; it may very well be that different circuits and/or cell types are involved but that both contribute to the same computation (albeit with different time constants or delays).

The latency (delay) of response onset is stimulus dependent (186) and is generally longer than offset latency (128). The current family of models cannot, however, be falsified by measurements of response latencies. Latency (delay) is different from the effective time constant (sluggishness). Latency may depend mostly on precortical processing and action potential conduction delays. For the simulations reported here, we assumed a particular form for the precortical temporal filter and negligible conduction delays. But the precortical filter and conduction delays could be changed without sacrificing the core idea that normalization arises from recurrent amplification, as expressed by Eqs. 1-6.

\section{Mechanisms}

We have presented a computational theory for what computations are performed by neural circuits in V1, not 
bioRxiv preprint doi: https://doi.org/10.1101/2020.03.22.002634; this version posted May 30, 2020. The copyright holder for this preprint (which was not certified by peer review) is the author/funder. All rights reserved. No reuse allowed without permission.

Dynamic Normalization

May 30, 2020

how they are implemented. But we can speculate about the underlying mechanisms:

- The circuit (Fig. 2) comprises an excitatory principal cell ( $y_{j}$, possibly layer 5 pyramidal cell), an inhibitory modulator cell ( $a_{j}$, presumably one or more of the inhibitory interneuron cell types) that may act via shunting, and another excitatory cell type $\left(u_{j}\right.$, possibly layer 2-3 pyramidal cell) that makes local recurrent connections. Each type of neuron performs a different dendritic computation (Eqs. 1, 5, and 6).

- The circuit also includes inhibitory interneurons (Fig. 2, small circles) to invert the sign of the LGN inputs and principal cells, corresponding to negative weights in the synaptic weight matrices $\mathbf{W}_{z x}$ and $\mathbf{W}_{\hat{y} y}$. These inhibitory neurons need not be 1-to-1 with their excitatory inputs, as drawn in the figure. Rather, each may compute a weighted sum of their inputs to contribute the terms in Eqs. 3-4 with negative weights.

- The responses of the principal cells (Eq. 1) may be implemented with a simplified biophysical (equivalent electrical circuit) model of a pyramidal cell $(80,123)$, in which the two terms of Eq. 1 are computed in separate dendritic compartments. The conductance of the first compartment determines the input gain and the synaptic current in that compartment is the input drive. The conductance and synaptic current in the second compartment correspond, respectively, to the recurrent gain and recurrent drive. The conductances and synaptic currents in each compartment may be controlled independently (6).

- The input drive is computed with positive and negative synaptic weights, i.e., both feedforward excitation and feedforward inhibition (Fig. 2, blue solid and dashed lines, respectively).

- The recurrent drive also involves both excitation and inhibition (Fig. 2, green solid and dashed lines, respectively; SI Appendix, Eq. S7), presumably via lateral connections within V1. These excitatory and inhibitory recurrent signals are both amplified by an amount that is controlled by the modulator cells, consistent with the experimental observation that surround suppression involves a decrease in both recurrent excitatory and recurrent inhibitory conductances (114).

- Some principal cells may share the same modulators (e.g., principal cells with the same RF and orientation preference but with different temporal phases; see SI Appendix for details), suggesting a much larger number of principal cells than modulator cells.

- The squaring nonlinearity (Eq. 2) may be approximated with a high threshold in combination with neural noise (187-189).

- The square roots in Eqs. 4-5 may be approximated by synaptic depression, which acts as a compressive nonlinearity because the probability of neurotransmitter release is lower at higher firing rates. Alternatively, the square roots in Eqs. 4-5 may be replaced by adding another cell type in the circuit (see SI Appendix, Eq. S39).

- The modulator cells with firing rates $a_{j}$ may act via shunting $(80,123)$, i.e., increasing conductance by a balanced increase in excitation and inhibition without changing the total synaptic current $(6,190)$. Such a conductance increase would, of course, further decrease the intrinsic time constant. Other mechanisms for multiplying/dividing neural signals may be substituted for shunting.

- The modulator cells may correspond to V1 parvalbumin-expressing (PV) inhibitory interneurons (191) and/ or somatostatin-expressing (SOM) inhibitory neurons (192). The modulator cell responses may depend in part on loops through higher visual cortical areas (193-196) and/or thalamocortical loops (197-204). The modulator cells are expected to have large RFs and broad orientation-selectivity (reflecting properties of the normalization pool), consistent with the response properties of SOM and PV neurons, respectively.

- Modulator cell responses $a_{j}$ depend on a product of $a_{j}$ with the square root of $u_{j}$ (Eq. 5); this may be computed with a synaptic current from $u_{j}$ and an intrinsic voltage-sensitive ion channel (205) such that conductance is inversely proportional to membrane depolarization $a_{j}$ (noting that firing rates $a_{j}$ are proportional to membrane depolarization).

- Eq. 6 comprises a summation over $w_{j k} y_{k} u_{k}$, each term of which may be computed in separate dendritic compartments.

Some of the effects of cross-orientation suppression may be due to feedforward (not recurrent) mechanisms, and the simulations here incorrectly ignored the fact that some of the normalization is inherited from the LGN inputs. Contrast saturation and rectification in LGN cells can largely account for the response suppression measured in cat primary visual cortex (206), and the responses of V1 neurons are suppressed by high temporal frequency stimuli that do not drive cortical responses (207). Consequently, cross-orientation suppression has been attributed to either precortical mechanisms (206), synaptic depression at the thalamocortical synapse (113), or fast feedforward inhibition via local interneurons within V1 (207), whereas feedback from higher visual cortical areas has been implicated in surround suppression (193-196). Nevertheless, few studies have addressed this question in macaque (63), and some evidence suggests that cortical circuits make an important contribution to 
bioRxiv preprint doi: https://doi.org/10.1101/2020.03.22.002634; this version posted May 30, 2020. The copyright holder for this preprint (which was not certified by peer review) is the author/funder. All rights reserved. No reuse allowed without permission.

Dynamic Normalization

May 30, 2020

cross-orientation suppression (43). As noted above, thalamocortical loops may contribute to the computation of the modulator cell responses, along with lateral connections within V1 and feedback connections from higher visual cortical areas. Regardless, there is consensus that some of the effects of normalization are computed with cortical circuits.

\section{Comparison with previous models}

The current theoretical framework is superior to both the original recurrent normalization model and alternative recurrent models of normalization (4-6, 39, 55, 113-117). First, none of the previous models converge exactly to the normalization equation (Eqs. 7-8) for arbitrary (non-negative) normalization weights. Although they may approximate weighted normalization, the extent to which the previous recurrent models fit the full range of experimental data is unknown. The current family of recurrent circuit models has a mathematically-tractable solution that equals weighted normalization. This has practical consequences, enabling us to derive closed-form expressions (Eqs. 7-10; see also SI Appendix) for making experimentally-testable predictions and for fitting data. Second, the current theoretical framework, unlike previous models, mimics the dynamics of V1 activity including slow onsets for low contrast stimuli, rapid onsets for high contrasts, and gamma oscillations for large, high-contrast, narrow-band stimuli. Third, most of the previous models do not rely on recurrent amplification to achieve normalization. Fourth, the current theoretical framework is applicable to diverse cognitive processes and neural systems (e.g., working memory and motor control), enabling us to use $\mathrm{V} 1$ as a model system for understanding the neural computations and circuits in many brain areas. Fifth, by virtue of being a generalization of LSTMs, the current theoretical framework can solve relatively sophisticated tasks.

The current family of models is similar in some respects to the inhibition stabilized network (ISN) (114) and the stabilized supralinear network (SSN) (116), but there are also crucial differences. All of these models include recurrent excitation that would be unstable if inhibition was absent or held fixed. All of them also include inhibitory stabilization, but the stabilizing inhibition in the current model is modulatory (multiplicative), unlike ISN and SSN in which inhibition is subtractive. Inhibitory stabilization, by itself, does not explain the phenomena associated with normalization. A linear recurrent model, that does not exhibit any of the nonlinear effects associated with normalization, may be stabilized by inhibition, i.e., such that it would be unstable if inhibition were removed or held fixed $(116,208)$. Normalization phenomena arise in the SSN model from a combination of amplification and inhibitory stabilization. SSN (116) and also earlier models $(4-6,55)$, amplify weak inputs more than strong inputs due to a power law relationship (e.g., half-squaring) between membrane depolarization and firing rate (187-189, 209). Removing the power function from SSN yields a linear model that is qualitatively different, in which responses increase in proportion to contrast (116). The current family of models also includes half-squaring, but it is not critical for normalization. Removing the squaring yields qualitatively similar phenomena; for example, the contrast-response function would be proportional to $c /(c+\sigma)$ rather than $c^{2} /\left(c^{2}+\sigma^{2}\right)$. Instead normalization in the current models relies on recurrent amplification via the product of recurrent gain and recurrent drive.

\section{Predictions}

The real value of this family of recurrent circuit models of normalization rests on whether it can push the field forward by making quantitative and testable predictions, leading to new experiments that may reveal novel phenomena. Some of these predictions are as follows.

- Our theoretical framework predicts that the effective time constant is contrast dependent (Eqs. 9-10); high contrast stimuli are integrated over much briefer periods of time (by a factor of $\sim 10 \mathrm{x}$ ) than low contrast stimuli. A functional advantage of doing so is to increase the signal-to-noise ratio (SNR) of responses evoked by a low contrast stimulus. Low contrasts evoke weak input drives with correspondingly low SNRs. Integrating these inputs over a long period of time (i.e., with a lowpass filter or local average) increases the SNR of the neural representation. This hypothesized difference in dynamics could be tested either electrophysiologically or psychophysically.

- We hypothesize a link between effective gain and effective time constant: effective time constant should increase with the square root of effective gain (Eq. 10). This is analogous to the previous shunting inhibition model of normalization $(6,55)$, but the prediction of that model was that both the gain and time constant change with intrinsic conductance, whereas the effective gain and time constant in the current family of models is a network effect, emerging from the recurrent amplification in the circuit. This hypothesized link may be tested either electrophysiologically or using psychophysical/behavioral methods (67).

- The link between effective gain and effective time constant is further constrained by the value of the input gain parameter $b_{0}$ (Eq. 10). The input gain of neurons in layer $4 \mathrm{C}$ (the input layer) may be estimated from intracellular measurements of membrane potential fluctuations with and without disabling cortical spikes (e.g., via optogenetics) as simulated in Figs. 3g,h. The input gain may also be manipulated with attention (e.g., 23).

- We predict a link between the intrinsic time constants and oscillation frequencies (Fig. 7e). In our simulations, oscillation frequency depended systematically on the values of the intrinsic time constants $\left(\tau_{v}, \tau_{a}\right.$, and $\left.\tau_{u}\right)$, 
bioRxiv preprint doi: https://doi.org/10.1101/2020.03.22.002634; this version posted May 30, 2020. The copyright holder for this preprint (which was not certified by peer review) is the author/funder. All rights reserved. No reuse allowed without permission.

Dynamic Normalization

May 30, 2020

and the input gain $\left(b_{0}\right)$. An experimental test of this prediction would involve manipulating the intrinsic time constant (i.e., the conductance) of a particular cell type in the circuit.

- The effects shown in Figs. 4d-f (increasing responsivity of both low and high temporal-frequencies with increasing contrast, and shifting response phases in opposite directions for temporal frequencies above and below the preferred temporal frequency) may be evident for neurons with narrow temporal-frequency tuning, e.g., perhaps direction-selective neurons in layer $4 b$.

\section{Acknowledgements}

\section{Funding: None}

Special thanks to Mike Landy, Adam Kohn, Jon Winawer, and Lyndon Duong for comments and discussion. 


\section{References}

1. D. J. Heeger, E. H. Adelson, Nonlinear Model of Cat Striate Cortex. Optics News 15, A-42 (1989).

2. D. G. Albrecht, W. S. Geisler, Motion sensitivity and the contrast-response function of simple cells in the visual cortex. Visual Neuroscience 7, 531-546 (1991).

3. D. J. Heeger, "Nonlinear model of neural responses in cat visual cortex" in Computational models of visual processing, M. S. Landy, J. A. Movshon, Eds. (MIT Press, Cambridge, MA, 1991), pp. 119-133.

4. D. J. Heeger, Normalization of cell responses in cat striate cortex. Vis Neurosci 9, 181-197 (1992).

5. D. J. Heeger, Modeling simple-cell direction selectivity with normalized, half- squared, linear operators. J Neurophysiol 70, 1885-1898 (1993).

6. M. Carandini, D. J. Heeger, Summation and division by neurons in primate visual cortex. Science 264, 1333-1336 (1994).

7. M. Carandini, D. J. Heeger, Normalization as a canonical neural computation. Nat Rev Neurosci 13, 51-62 (2011).

8. E. P. Simoncelli, D. J. Heeger, A model of neuronal responses in visual area MT. Vision Res 38, 743-761 (1998).

9. J. H. Reynolds, L. Chelazzi, R. Desimone, Competitive mechanisms subserve attention in macaque areas V2 and V4. J Neurosci 19, 1736-1753 (1999).

10. O. Schwartz, E. P. Simoncelli, Natural signal statistics and sensory gain control. Nat Neurosci 4, 819-825 (2001).

11. O. Schwartz, E. P. Simoncelli (2001) Natural sound statistics and divisive normalization in the auditory system. in Advances in neural information processing systems, pp 166-172.

12. M. J. Wainwright, O. Schwartz, E. P. Simoncelli, "Natural Image Statistics and Divisive Normalization" in Probabilistic Models of the Brain: Perception and Neural Function, R. P. N. Rao, B. A. Olshausen, M. S. Lewicki, Eds. (MIT Press, 2002), pp. 203-.

13. D. Zoccolan, D. D. Cox, J. J. DiCarlo, Multiple object response normalization in monkey inferotemporal cortex. J Neurosci 25, 8150-8164 (2005).

14. N. C. Rust, V. Mante, E. P. Simoncelli, J. A. Movshon, How MT cells analyze the motion of visual patterns. Nature neuroscience 9, 1421-1431 (2006).

15. T. Sugihara, M. D. Diltz, B. B. Averbeck, L. M. Romanski, Integration of auditory and visual communication information in the primate ventrolateral prefrontal cortex. J Neurosci 26, 11138-11147 (2006).

16. M. Avillac, S. Ben Hamed, J. R. Duhamel, Multisensory integration in the ventral intraparietal area of the macaque monkey. J Neurosci 27, 1922-1932 (2007).

17. M. B. Ahrens, J. F. Linden, M. Sahani, Nonlinearities and contextual influences in auditory cortical responses modeled with multilinear spectrotemporal methods. J Neurosci 28, 1929-1942 (2008).

18. G. M. Boynton, A framework for describing the effects of attention on visual responses. Vision Res 49, 1129-1143 (2009).

19. L. Busse, A. R. Wade, M. Carandini, Representation of concurrent stimuli by population activity in visual cortex. Neuron 64, 931-942 (2009).

20. S. V. David, N. Mesgarani, J. B. Fritz, S. A. Shamma, Rapid synaptic depression explains nonlinear modulation of spectro-temporal tuning in primary auditory cortex by natural stimuli. $J \mathrm{Neu}$ rosci 29, 3374-3386 (2009). 
21. J. Lee, J. H. Maunsell, A normalization model of attentional modulation of single unit responses. PLOS ONE 4, e4651 (2009).

22. F. Moradi, D. J. Heeger, Inter-ocular contrast normalization in human visual cortes. Journal of vision 9(3), 1-22 (2009).

23. J. H. Reynolds, D. J. Heeger, The normalization model of attention. Neuron 61, 168-185 (2009).

24. K. A. Sundberg, J. F. Mitchell, J. H. Reynolds, Spatial attention modulates center-surround interactions in macaque visual area v4. Neuron 61, 952-963 (2009).

25. K. Herrmann, L. Montaser-Kouhsari, M. Carrasco, D. J. Heeger, When size matters: attention affects performance by contrast or response gain. Nat Neurosci 13, 1554-1559 (2010).

26. P. S. Khayat, R. Niebergall, J. C. Martinez-Trujillo, Attention differentially modulates similar neuronal responses evoked by varying contrast and direction stimuli in area MT. J Neurosci 30, 2188-2197 (2010).

27. J. Lee, J. H. Maunsell, Attentional modulation of MT neurons with single or multiple stimuli in their receptive fields. J Neurosci 30, 3058-3066 (2010).

28. S. R. Olsen, V. Bhandawat, R. I. Wilson, Divisive normalization in olfactory population codes. Neuron 66, 287-299 (2010).

29. G. J. Brouwer, D. J. Heeger, Cross-orientation suppression in human visual cortex. Journal of neurophysiology 106, 2108-2119 (2011).

30. K. Louie, L. E. Grattan, P. W. Glimcher, Reward value-based gain control: divisive normalization in parietal cortex. The Journal of neuroscience : the official journal of the Society for Neuroscience 31, 10627-10639 (2011).

31. T. Ohshiro, D. E. Angelaki, G. C. Deangelis, A normalization model of multisensory integration. Nature neuroscience 14, 775-782 (2011).

32. N. C. Rabinowitz, B. D. Willmore, J. W. Schnupp, A. J. King, Contrast gain control in auditory cortex. Neuron 70, 1178-1191 (2011).

33. T. Lochmann, U. A. Ernst, S. Deneve, Perceptual inference predicts contextual modulations of sensory responses. J Neurosci 32, 4179-4195 (2012).

34. K. Louie, M. W. Khaw, P. W. Glimcher, Normalization is a general neural mechanism for contextdependent decision making. Proc Natl Acad Sci U S A 110, 6139-6144 (2013).

35. S. Ray, A. M. Ni, J. H. Maunsell, Strength of gamma rhythm depends on normalization. PLoS Biol 11, e1001477 (2013).

36. O. Schwartz, R. Coen-Cagli, Visual attention and flexible normalization pools. J Vis 13 (2013).

37. M. M. Diehl, L. M. Romanski, Responses of prefrontal multisensory neurons to mismatching faces and vocalizations. J Neurosci 34, 11233-11243 (2014).

38. Y. Hara, F. Pestilli, J. L. Gardner, Differing effects of attention in single-units and populations are well predicted by heterogeneous tuning and the normalization model of attention. Frontiers in computational neuroscience 8, 12 (2014).

39. K. Louie, T. LoFaro, R. Webb, P. W. Glimcher, Dynamic divisive normalization predicts timevarying value coding in decision-related circuits. J Neurosci 34, 16046-16057 (2014).

40. T. K. Sato, M. Hausser, M. Carandini, Distal connectivity causes summation and division across mouse visual cortex. Nat Neurosci 17, 30-32 (2014).

41. S. G. Solomon, A. Kohn, Moving sensory adaptation beyond suppressive effects in single neurons. Curr Biol 24, R1012-1022 (2014).

42. G. J. Brouwer, V. Arnedo, S. Offen, D. J. Heeger, A. C. Grant, Normalization in human somatosensory cortex. J Neurophysiol 114, 2588-2599 (2015). 
43. J. J. Nassi, M. C. Avery, A. H. Cetin, A. W. Roe, J. H. Reynolds, Optogenetic Activation of Normalization in Alert Macaque Visual Cortex. Neuron 86, 1504-1517 (2015).

44. M. Snow, R. Coen-Cagli, O. Schwartz, Specificity and timescales of cortical adaptation as inferences about natural movie statistics. J Vis 16 (2016).

45. B. E. Verhoef, J. H. Maunsell, Attention operates uniformly throughout the classical receptive field and the surround. Elife 5 (2016).

46. Z. M. Westrick, D. J. Heeger, M. S. Landy, Pattern Adaptation and Normalization Reweighting. J Neurosci 36, 9805-9816 (2016).

47. M. W. Khaw, P. W. Glimcher, K. Louie, Normalized value coding explains dynamic adaptation in the human valuation process. Proc Natl Acad Sci U S A 114, 12696-12701 (2017).

48. A. M. Ni, J. H. R. Maunsell, Spatially tuned normalization explains attention modulation variance within neurons. J Neurophysiol 118, 1903-1913 (2017).

49. T. Ohshiro, D. E. Angelaki, G. C. DeAngelis, A Neural Signature of Divisive Normalization at the Level of Multisensory Integration in Primate Cortex. Neuron 95, 399-411 e398 (2017).

50. B. E. Verhoef, J. H. R. Maunsell, Attention-related changes in correlated neuronal activity arise from normalization mechanisms. Nat Neurosci 20, 969-977 (2017).

51. A. Aschner, S. G. Solomon, M. S. Landy, D. J. Heeger, A. Kohn, Temporal Contingencies Determine Whether Adaptation Strengthens or Weakens Normalization. J Neurosci 38, 10129-10142 (2018).

52. J. Zhou, N. C. Benson, K. N. Kay, J. Winawer, Compressive Temporal Summation in Human Visual Cortex. J Neurosci 38, 691-709 (2018).

53. M. C. Morrone, D. C. Burr, L. Maffei, Functional implications of cross-orientation inhibition of cortical visual cells. Proceedings of the Royal Society of London, B 216, 335-354 (1982).

54. M. C. Morrone, D. C. Burr, Evidence for the existence and development of visual inhibition in humans. Nature 321, 235-237 (1986).

55. M. Carandini, D. J. Heeger, J. A. Movshon, Linearity and normalization in simple cells of the macaque primary visual cortex. J Neurosci 17, 8621-8644 (1997).

56. D. J. Tolhurst, D. J. Heeger, Comparison of contrast-normalization and threshold models of the responses of simple cells in cat striate cortex. Vis Neurosci 14, 293-309 (1997).

57. D. J. Tolhurst, D. J. Heeger, Contrast normalization and a linear model for the directional selectivity of simple cells in cat striate cortex. Vis Neurosci 14, 19-25 (1997).

58. M. P. Sceniak, D. L. Ringach, M. J. Hawken, R. Shapley, Contrast's effect on spatial summation by macaque V1 neurons. Nat Neurosci 2, 733-739 (1999).

59. M. P. Sceniak, M. J. Hawken, R. Shapley, Visual spatial characterization of macaque V1 neurons. J Neurophysiol 85, 1873-1887. (2001).

60. J. R. Cavanaugh, W. Bair, J. A. Movshon, Nature and interaction of signals from the receptive field center and surround in macaque V1 neurons. J Neurophysiol 88, 2530-2546. (2002).

61. J. R. Cavanaugh, W. Bair, J. A. Movshon, Selectivity and spatial distribution of signals from the receptive field surround in macaque V1 neurons. J Neurophysiol 88, 2547-2556. (2002).

62. B. Zenger-Landolt, D. J. Heeger, Response suppression in V1 agrees with psychophysics of surround masking. J Neurosci 23, 6884-6893 (2003).

63. M. A. Smith, W. Bair, J. A. Movshon, Dynamics of suppression in macaque primary visual cortex. J Neurosci 26, 4826-4834 (2006).

64. J. M. Foley, Human luminance pattern-vision mechanisms: masking experiments require a new model. J Opt Soc Am A 11, 1710-1719. (1994). 
65. J. Xing, D. J. Heeger, Center-surround interactions in foveal and peripheral vision. Vision Res 40, 3065-3072 (2000).

66. J. Xing, D. J. Heeger, Measurement and modeling of center-surround suppression and enhancement. Vision Res 41, 571-583. (2001).

67. Y. Petrov, M. Carandini, S. McKee, Two distinct mechanisms of suppression in human vision. $J$ Neurosci 25, 8704-8707 (2005).

68. H. Yiltiz, D. J. Heeger, M. S. Landy, Contingent adaptation in masking and surround suppression. Vision Res 166, 72-80 (2020).

69. A. B. Bonds, Role of inhibition in the specification of orientation selectivity of cells in the cat striate cortex. Vis Neurosci 2, 41-55 (1989).

70. G. C. DeAngelis, J. G. Robson, I. Ohzawa, R. D. Freeman, Organization of suppression in receptive fields of neurons in cat visual cortex. J Neurophysiol 68, 144-163 (1992).

71. G. C. DeAngelis, R. D. Freeman, I. Ohzawa, Length and width tuning of neurons in the cat's primary visual cortex. J Neurophysiol 71, 347-374 (1994).

72. W. Bair, J. R. Cavanaugh, J. A. Movshon, Time course and time-distance relationships for surround suppression in macaque V1 neurons. J Neurosci 23, 7690-7701 (2003).

73. M. Carandini, D. J. Heeger, Normalization as a canonical neural computation. Nat Rev Neurosci 13, 51-62 (2011).

74. D. J. Heeger, E. P. Simoncelli, J. A. Movshon, Computational models of cortical visual processing. Proc Natl Acad Sci U S A 93, 623-627 (1996).

75. E. P. Simoncelli, "Local analysis of visual motion" in The Visual Neurosciences, L. M. Chalupa, J. S. Werner, Eds. (MIT Press, Cambridge, MA, 2003), pp. 1616-1623.

76. J. M. Beck, P. E. Latham, A. Pouget, Marginalization in neural circuits with divisive normalization. J Neurosci 31, 15310-15319 (2011).

77. D. L. Ringach, Population coding under normalization. Vision Res S0042-6989(09)00555-0 [pii] 10.1016/j.visres.2009.12.007 (2009).

78. S. Lyu, E. P. Simoncelli, Nonlinear extraction of independent components of natural images using radial gaussianization. Neural Comput 21, 1485-1519 (2009).

79. S. X. Luo, R. Axel, L. F. Abbott, Generating sparse and selective third-order responses in the olfactory system of the fly. Proc Natl Acad Sci U S A 107, 10713-10718 (2010).

80. D. J. Heeger, W. E. Mackey, Oscillatory recurrent gated neural integrator circuits (ORGaNICs), a unifying theoretical framework for neural dynamics. Proc Natl Acad Sci U S A 116, 22783-22794 (2019).

81. J. L. Ba, J. R. Kiros, G. E. Hinton, Layer normalization. arXiv preprint arXiv:1607.06450 (2016).

82. M. Ren, R. Liao, R. Urtasun, F. H. Sinz, R. S. Zemel, Normalizing the normalizers: Comparing and extending network normalization schemes. arXiv preprint arXiv:1611.04520 (2016).

83. Y. F. Sit, Y. Chen, W. S. Geisler, R. Miikkulainen, E. Seidemann, Complex dynamics of V1 population responses explained by a simple gain-control model. Neuron 64, 943-956 (2009).

84. R. A. Holub, M. Morton-Gibson, Response of Visual Cortical Neurons of the cat to moving sinusoidal gratings: response-contrast functions and spatiotemporal interactions. J Neurophysiol 46, 1244-1259 (1981).

85. A. F. Dean, D. J. Tolhurst, Factors influencing the temporal phase of response to bar and grating stimuli for simple cells in the cat striate cortex. Experimental Brain Research 62, 143-151 (1986).

86. D. G. Albrecht, Visual cortex neurons in monkey and cat: Effect of contrast on the spatial and temporal phase transfer functions. Visual Neuroscience 12, 1191-1210 (1995). 
87. M. A. Gieselmann, A. Thiele, Comparison of spatial integration and surround suppression characteristics in spiking activity and the local field potential in macaque V1. Eur J Neurosci 28, 447459 (2008).

88. D. Hermes, N. Petridou, K. N. Kay, J. Winawer, An image-computable model for the stimulus selectivity of gamma oscillations. Elife 8 (2019).

89. C. Kayser, R. F. Salazar, P. Konig, Responses to natural scenes in cat V1. J Neurophysiol 90, 1910-1920 (2003).

90. J. A. Henrie, R. Shapley, LFP power spectra in V1 cortex: the graded effect of stimulus contrast. J Neurophysiol 94, 479-490 (2005).

91. Z. Zhou, M. R. Bernard, A. B. Bonds, Deconstruction of spatial integrity in visual stimulus detected by modulation of synchronized activity in cat visual cortex. J Neurosci 28, 3759-3768 (2008).

92. B. Lima, W. Singer, N. H. Chen, S. Neuenschwander, Synchronization dynamics in response to plaid stimuli in monkey V1. Cereb Cortex 20, 1556-1573 (2010).

93. S. Ray, J. H. Maunsell, Differences in gamma frequencies across visual cortex restrict their possible use in computation. Neuron 67, 885-896 (2010).

94. M. J. Bartolo et al., Stimulus-induced dissociation of neuronal firing rates and local field potential gamma power and its relationship to the resonance blood oxygen level-dependent signal in macaque primary visual cortex. Eur J Neurosci 34, 1857-1870 (2011).

95. X. Jia, A. Kohn, Gamma rhythms in the brain. PLoS Biol 9, e1001045 (2011).

96. X. Jia, M. A. Smith, A. Kohn, Stimulus selectivity and spatial coherence of gamma components of the local field potential. J Neurosci 31, 9390-9403 (2011).

97. S. Ray, J. H. Maunsell, Different origins of gamma rhythm and high-gamma activity in macaque visual cortex. PLoS Biol 9, e1000610 (2011).

98. X. Jia, D. Xing, A. Kohn, No consistent relationship between gamma power and peak frequency in macaque primary visual cortex. J Neurosci 33, 17-25 (2013).

99. D. Hermes, K. J. Miller, B. A. Wandell, J. Winawer, Stimulus Dependence of Gamma Oscillations in Human Visual Cortex. Cereb Cortex 25, 2951-2959 (2015).

100. M. W. Self et al., The Effects of Context and Attention on Spiking Activity in Human Early Visual Cortex. PLoS Biol 14, e1002420 (2016).

101. E. Bartoli et al., Functionally Distinct Gamma Range Activity Revealed by Stimulus Tuning in Human Visual Cortex. Curr Biol 29, 3345-3358 e3347 (2019).

102. N. M. Brunet, P. Fries, Human visual cortical gamma reflects natural image structure. Neurolmage 200, 635-643 (2019).

103. H. Adesnik, M. Scanziani, Lateral competition for cortical space by layer-specific horizontal circuits. Nature 464, 1155-1160 (2010).

104. T. K. Sato, B. Haider, M. Hausser, M. Carandini, An excitatory basis for divisive normalization in visual cortex. Nat Neurosci 19, 568-570 (2016).

105. H. Adesnik, Synaptic Mechanisms of Feature Coding in the Visual Cortex of Awake Mice. Neuron 95, 1147-1159 e1144 (2017).

106. K. A. Bolding, K. M. Franks, Recurrent cortical circuits implement concentration-invariant odor coding. Science 361 (2018).

107. R. J. Douglas, C. Koch, M. Mahowald, K. A. Martin, H. H. Suarez, Recurrent excitation in neocortical circuits. Science 269, 981-985. (1995).

108. E. M. Callaway, Local circuits in primary visual cortex of the macaque monkey. Annu Rev Neurosci 21, 47-74 (1998). 
bioRxiv preprint doi: https://doi.org/10.1101/2020.03.22.002634; this version posted May 30, 2020. The copyright holder for this preprint (which was not certified by peer review) is the author/funder. All rights reserved. No reuse allowed without permission.

Dynamic Normalization

May 30, 2020

109. Y. Yoshimura, J. L. Dantzker, E. M. Callaway, Excitatory cortical neurons form fine-scale functional networks. Nature 433, 868-873 (2005).

110. R. J. Douglas, K. A. Martin, Recurrent neuronal circuits in the neocortex. Curr Biol 17, R496-500 (2007).

111. C. Kapfer, L. L. Glickfeld, B. V. Atallah, M. Scanziani, Supralinear increase of recurrent inhibition during sparse activity in the somatosensory cortex. Nat Neurosci 10, 743-753 (2007).

112. S. Peron et al., Recurrent interactions in local cortical circuits. Nature 579, 256-259 (2020).

113. M. Carandini, D. J. Heeger, W. Senn, A synaptic explanation of suppression in visual cortex. J Neurosci 22, 10053-10065. (2002).

114. H. Ozeki, I. M. Finn, E. S. Schaffer, K. D. Miller, D. Ferster, Inhibitory stabilization of the cortical network underlies visual surround suppression. Neuron 62, 578-592 (2009).

115. T. Brosch, H. Neumann, Computing with a canonical neural circuits model with pool normalization and modulating feedback. Neural Comput 26, 2735-2789 (2014).

116. D. B. Rubin, S. D. Van Hooser, K. D. Miller, The stabilized supralinear network: a unifying circuit motif underlying multi-input integration in sensory cortex. Neuron 85, 402-417 (2015).

117. E. Koch, J. Jin, J. M. Alonso, Q. Zaidi, Functional implications of orientation maps in primary visual cortex. Nat Commun 7, 13529 (2016).

118. G. Sperling, M. M. Sondhi, Model for visual luminance discrimination and flicker detection. Journal of the Optical Society of America 58, 1133-1145 (1968).

119. S. Grossberg, Contour enhancement, short-term memory, and constancies in reverberating neural networks. Stud App Math 52, 217-257 (1973).

120. S. Grossberg, Adaptive pattern classification and universal recoding: I. Parallel development and coding of neural feature detectors. Biol Cybern 23, 121-134 (1976).

121. D. J. Heeger, K. O. Zemlianova, Dynamic Normalization. bioRxiv 10.1101/2020.03.22.002634, 2020.2003.2022.002634 (2020).

122. D. J. Heeger, K. O. Zemlianova (2020) Supplemental Material for "Dynamic Normalization". (NYU Faculty Digial Archive, https://archive.nyu.edu/handle/2451/61045).

123. D. J. Heeger, W. E. Mackey, ORGaNICs: A Theory of Working Memory in Brains and Machines. arXiv preprint arXiv:1803.06288 (2018).

124. J. R. Muller, A. B. Metha, J. Krauskopf, P. Lennie, Information conveyed by onset transients in responses of striate cortical neurons. J Neurosci 21, 6978-6990. (2001).

125. D. G. Albrecht, W. S. Geisler, R. A. Frazor, A. M. Crane, Visual cortex neurons of monkeys and cats: temporal dynamics of the contrast response function. J Neurophysiol 88, 888-913 (2002).

126. D. J. T. N. S. Walker, I. D. Thompson, A. F. Dean, Nonlinearities of temporal summation in neurones in area 17 of the cat. Experimental Brain Research 38, 431-435 (1980).

127. A. F. Dean, D. J. Tolhurst, On the distinctness of simple and complex cells in the visual cortex of the cat. J Physiol 344, 305-325 (1983).

128. W. Bair, J. R. Cavanaugh, M. A. Smith, J. A. Movshon, The timing of response onset and offset in macaque visual neurons. J Neurosci 22, 3189-3205. (2002).

129. S. P. Burns, D. Xing, R. M. Shapley, Comparisons of the dynamics of local field potential and multiunit activity signals in macaque visual cortex. $J$ Neurosci 30, 13739-13749 (2010).

130. J. R. Muller, A. B. Metha, J. Krauskopf, P. Lennie, Rapid adaptation in visual cortex to the structure of images. Science 285, 1405-1408. (1999).

131. D. L. Ringach, M. J. Hawken, R. Shapley, Dynamics of orientation tuning in macaque primary visual cortex. Nature 387, 281-284 (1997). 
132. D. Ferster, S. Chung, H. S. Wheat, Orientation selectivity of synaptic input from lateral geniculate nucleus to simple cells of cat visual cortex. Nature 380, 249-252 (1996).

133. S. Chung, D. Ferster, Strength and orientation tuning of the thalamic input to simple cells revealed by electrically evoked cortical suppression. Neuron 20, 1177-1189 (1998).

134. K. Reinhold, A. D. Lien, M. Scanziani, Distinct recurrent versus afferent dynamics in cortical visual processing. Nat Neurosci 18, 1789-1797 (2015).

135. A. D. Lien, M. Scanziani, Cortical direction selectivity emerges at convergence of thalamic synapses. Nature 558, 80-86 (2018).

136. K. Kang, M. Shelley, J. A. Henrie, R. Shapley, LFP spectral peaks in V1 cortex: network resonance and cortico-cortical feedback. J Comput Neurosci 29, 495-507 (2010).

137. S. Hochreiter, J. Schmidhuber, Long short-term memory. Neural Comput 9, 1735-1780 (1997).

138. A. Graves, Generating sequences with recurrent neural networks. arXiv preprint arXiv: 1308.0850 (2013).

139. A. Graves, A.-r. Mohamed, G. Hinton (2013) Speech recognition with deep recurrent neural networks. in Acoustics, speech and signal processing (icassp), 2013 ieee international conference on (IEEE), pp 6645-6649.

140. K. Cho et al., Learning phrase representations using RNN encoder-decoder for statistical machine translation. arXiv preprint arXiv:1406.1078 (2014).

141. I. Sutskever, O. Vinyals, Q. V. Le (2014) Sequence to sequence learning with neural networks. in Advances in neural information processing systems, pp 3104-3112.

142. D. J. Heeger, Theory of cortical function. Proc Natl Acad Sci U S A 114, 1773-1782 (2017).

143. R. Eckhorn et al., Coherent oscillations: a mechanism of feature linking in the visual cortex? Multiple electrode and correlation analyses in the cat. Biol Cybern 60, 121-130 (1988).

144. C. M. Gray, P. Konig, A. K. Engel, W. Singer, Oscillatory responses in cat visual cortex exhibit inter-columnar synchronization which reflects global stimulus properties. Nature 338, 334-337 (1989).

145. W. Singer, Neuronal synchrony: a versatile code for the definition of relations? Neuron 24, 49-65, 111-125. (1999).

146. A. K. Engel, P. Fries, W. Singer, Dynamic predictions: oscillations and synchrony in top-down processing. Nat Rev Neurosci 2, 704-716 (2001).

147. H. Tiitinen et al., Selective attention enhances the auditory $40-\mathrm{Hz}$ transient response in humans. Nature 364, 59-60 (1993).

148. P. Fries, J. H. Reynolds, A. E. Rorie, R. Desimone, Modulation of oscillatory neuronal synchronization by selective visual attention. Science 291, 1560-1563. (2001).

149. E. Salinas, T. J. Sejnowski, Correlated neuronal activity and the flow of neural information. Nat Rev Neurosci 2, 539-550 (2001).

150. G. Deco, A. Thiele, Attention: oscillations and neuropharmacology. Eur J Neurosci 30, 347-354 (2009).

151. M. Abeles, Role of the cortical neuron: integrator or coincidence detector? Isr J Med Sci 18, 8392 (1982).

152. P. Konig, A. K. Engel, W. Singer, Integrator or coincidence detector? The role of the cortical neuron revisited. Trends Neurosci 19, 130-137 (1996).

153. F. Varela, J. P. Lachaux, E. Rodriguez, J. Martinerie, The brainweb: phase synchronization and large-scale integration. Nat Rev Neurosci 2, 229-239 (2001).

154. P. Fries, A mechanism for cognitive dynamics: neuronal communication through neuronal coherence. Trends Cogn Sci 9, 474-480 (2005). 
Dynamic Normalization

May 30, 2020

155. C. Borgers, N. J. Kopell, Gamma oscillations and stimulus selection. Neural Comput 20, 383-414 (2008).

156. P. Fries, Neuronal gamma-band synchronization as a fundamental process in cortical computation. Annu Rev Neurosci 32, 209-224 (2009).

157. G. G. Gregoriou, S. J. Gotts, H. Zhou, R. Desimone, High-frequency, long-range coupling between prefrontal and visual cortex during attention. Science 324, 1207-1210 (2009).

158. T. Akam, D. M. Kullmann, Oscillations and filtering networks support flexible routing of information. Neuron 67, 308-320 (2010).

159. S. P. Burns, D. Xing, M. J. Shelley, R. M. Shapley, Searching for autocoherence in the cortical network with a time-frequency analysis of the local field potential. $J$ Neurosci 30, 4033-4047 (2010).

160. M. Chalk et al., Attention reduces stimulus-driven gamma frequency oscillations and spike field coherence in V1. Neuron 66, 114-125 (2010).

161. S. P. Burns, D. Xing, R. M. Shapley, Is gamma-band activity in the local field potential of V1 cortex a "clock" or filtered noise? J Neurosci 31, 9658-9664 (2011).

162. D. Xing et al., Stochastic generation of gamma-band activity in primary visual cortex of awake and anesthetized monkeys. J Neurosci 32, 13873-13880a (2012).

163. D. Hermes, K. J. Miller, B. A. Wandell, J. Winawer, Gamma oscillations in visual cortex: the stimulus matters. Trends Cogn Sci 19, 57-58 (2015).

164. V. Shirhatti, S. Ray, Long-wavelength (reddish) hues induce unusually large gamma oscillations in the primate primary visual cortex. Proc Natl Acad Sci U S A 115, 4489-4494 (2018).

165. L. M. Hurvich, D. Jameson, An opponent-process theory of color vision. Psychol Rev 64, Part 1, 384-404 (1957).

166. A. M. Derrington, J. Krauskopf, P. Lennie, Chromatic mechanisms in lateral geniculate nucleus of macaque. J Physiol 357, 241-265 (1984).

167. P. K. Kaiser, R. M. Boynton, Human color vision. (1996).

168. N. Brunel, X. J. Wang, What determines the frequency of fast network oscillations with irregular neural discharges? I. Synaptic dynamics and excitation-inhibition balance. J Neurophysio/ $\mathbf{9 0}$, 415-430 (2003).

169. S. Keeley, A. Byrne, A. Fenton, J. Rinzel, Firing rate models for gamma oscillations. J Neurophysiol 121, 2181-2190 (2019).

170. W. J. Freeman, Relations between unit activity and evoked potentials in prepyriform cortex of cats. J Neurophysiol 31, 337-348 (1968).

171. J. M. Horowitz, Evoked activity of single units and neural populations in the hippocampus of the cat. Electroencephalogr Clin Neurophysiol 32, 227-240 (1972).

172. H. R. Wilson, J. D. Cowan, Excitatory and inhibitory interactions in localized populations of model neurons. Biophys J 12, 1-24 (1972).

173. L. S. Leung, Nonlinear feedback model of neuronal populations in hippocampal CAl region. $J$ Neurophysiol 47, 845-868 (1982).

174. M. A. Whittington, R. D. Traub, J. G. Jefferys, Synchronized oscillations in interneuron networks driven by metabotropic glutamate receptor activation. Nature 373, 612-615 (1995).

175. P. Bush, T. Sejnowski, Inhibition synchronizes sparsely connected cortical neurons within and between columns in realistic network models. J Comput Neurosci 3, 91-110 (1996).

176. R. D. Traub, M. A. Whittington, I. M. Stanford, J. G. Jefferys, A mechanism for generation of long-range synchronous fast oscillations in the cortex. Nature 383, 621-624 (1996). 
bioRxiv preprint doi: https://doi.org/10.1101/2020.03.22.002634; this version posted May 30, 2020. The copyright holder for this preprint (which was not certified by peer review) is the author/funder. All rights reserved. No reuse allowed without permission.

Dynamic Normalization

May 30, 2020

177. R. D. Traub, J. G. Jefferys, M. A. Whittington, Simulation of gamma rhythms in networks of interneurons and pyramidal cells. J Comput Neurosci 4, 141-150 (1997).

178. M. A. Whittington, R. D. Traub, N. Kopell, B. Ermentrout, E. H. Buhl, Inhibition-based rhythms: experimental and mathematical observations on network dynamics. Int J Psychophysiol 38, 315-336 (2000).

179. P. Tiesinga, T. J. Sejnowski, Cortical enlightenment: are attentional gamma oscillations driven by ING or PING? Neuron 63, 727-732 (2009).

180. X. J. Wang, Neurophysiological and computational principles of cortical rhythms in cognition. Physiol Rev 90, 1195-1268 (2010).

181. P. H. Tiesinga, J. M. Fellous, E. Salinas, J. V. Jose, T. J. Sejnowski, Inhibitory synchrony as a mechanism for attentional gain modulation. Journal of physiology, Paris 98, 296-314 (2004).

182. K. Taylor, S. Mandon, W. A. Freiwald, A. K. Kreiter, Coherent oscillatory activity in monkey area v4 predicts successful allocation of attention. Cereb Cortex 15, 1424-1437 (2005).

183. C. Borgers, S. Epstein, N. J. Kopell, Gamma oscillations mediate stimulus competition and attentional selection in a cortical network model. Proc Natl Acad Sci U S A 105, 18023-18028 (2008).

184. P. Fries, T. Womelsdorf, R. Oostenveld, R. Desimone, The effects of visual stimulation and selective visual attention on rhythmic neuronal synchronization in macaque area V4. J Neurosci 28, 4823-4835 (2008).

185. M. Vinck, T. Womelsdorf, E. A. Buffalo, R. Desimone, P. Fries, Attentional modulation of cellclass-specific gamma-band synchronization in awake monkey area v4. Neuron 80, 1077-1089 (2013).

186. T. J. Gawne, T. W. Kjaer, B. J. Richmond, Latency: another potential code for feature binding in striate cortex. J Neurophysiol 76, 1356-1360 (1996).

187. J. S. Anderson, I. Lampl, D. C. Gillespie, D. Ferster, The contribution of noise to contrast invariance of orientation tuning in cat visual cortex. Science 290, 1968-1972 (2000).

188. M. Carandini, D. Ferster, Membrane potential and firing rate in cat primary visual cortex. $J$ Neurosci 20, 470-484 (2000).

189. K. D. Miller, T. W. Troyer, Neural noise can explain expansive, power-law nonlinearities in neural response functions. J Neurophysiol 87, 653-659 (2002).

190. F. S. Chance, L. F. Abbott, A. D. Reyes, Gain modulation from background synaptic input. Neuron 35, 773-782 (2002).

191. B. V. Atallah, W. Bruns, M. Carandini, M. Scanziani, Parvalbumin-expressing interneurons linearly transform cortical responses to visual stimuli. Neuron 73, 159-170 (2012).

192. H. Adesnik, W. Bruns, H. Taniguchi, Z. J. Huang, M. Scanziani, A neural circuit for spatial summation in visual cortex. Nature 490, 226-231 (2012).

193. A. Angelucci, J. B. Levitt, J. S. Lund, Anatomical origins of the classical receptive field and modulatory surround field of single neurons in macaque visual cortical area V1. Prog Brain Res 136, 373-388 (2002).

194. A. Angelucci et al., Circuits for local and global signal integration in primary visual cortex. $J$ Neurosci 22, 8633-8646. (2002).

195. J. J. Nassi, S. G. Lomber, R. T. Born, Corticocortical feedback contributes to surround suppression in V1 of the alert primate. J Neurosci 33, 8504-8517 (2013).

196. J. J. Nassi, C. Gomez-Laberge, G. Kreiman, R. T. Born, Corticocortical feedback increases the spatial extent of normalization. Front Syst Neurosci 8, 105 (2014).

197. N. D. Schiff et al., Behavioural improvements with thalamic stimulation after severe traumatic brain injury. Nature 448, 600-603 (2007). 
bioRxiv preprint doi: https://doi.org/10.1101/2020.03.22.002634; this version posted May 30, 2020. The copyright holder for this preprint (which was not certified by peer review) is the author/funder. All rights reserved. No reuse allowed without permission.

Dynamic Normalization

May 30, 2020

198. B. B. Theyel, D. A. Llano, S. M. Sherman, The corticothalamocortical circuit drives higher-order cortex in the mouse. Nat Neurosci 13, 84-88 (2010).

199. Y. B. Saalmann, M. A. Pinsk, L. Wang, X. Li, S. Kastner, The pulvinar regulates information transmission between cortical areas based on attention demands. Science 337, 753-756 (2012).

200. Z. V. Guo et al., Maintenance of persistent activity in a frontal thalamocortical loop. Nature 545, 181-186 (2017).

201. L. I. Schmitt et al., Thalamic amplification of cortical connectivity sustains attentional control. Nature 545, 219-223 (2017).

202. R. V. Rikhye, A. Gilra, M. M. Halassa, Thalamic regulation of switching between cortical representations enables cognitive flexibility. Nat Neurosci 21, 1753-1763 (2018).

203. R. V. Rikhye, R. D. Wimmer, M. M. Halassa, Toward an Integrative Theory of Thalamic Function. Annual review of neuroscience 41, 163-183 (2018).

204. M. J. Redinbaugh et al., Thalamus Modulates Consciousness via Layer-Specific Control of Cortex. Neuron 10.1016/j.neuron.2020.01.005 (2020).

205. B. Li, B. N. Routh, D. Johnston, E. Seidemann, N. J. Priebe, Voltage-Gated Intrinsic Conductances Shape the Input-Output Relationship of Cortical Neurons in Behaving Primate V1. Neuron 10.1016/j.neuron.2020.04.001 (2020).

206. N. J. Priebe, D. Ferster, Mechanisms underlying cross-orientation suppression in cat visual cortex. Nat Neurosci 9, 552-561 (2006).

207. T. C. Freeman, S. Durand, D. C. Kiper, M. Carandini, Suppression without inhibition in visual cortex. Neuron 35, 759-771 (2002).

208. M. V. Tsodyks, W. E. Skaggs, T. J. Sejnowski, B. L. McNaughton, Paradoxical effects of external modulation of inhibitory interneurons. J Neurosci 17, $4382-4388$ (1997).

209. D. J. Heeger, Half-squaring in responses of cat striate cells. Vis Neurosci 9, 427-443 (1992). 


\section{Supplemental Information for Dynamic Normalization}

David J. Heeger and Klavdia O. Zemlianova

Corresponding author: David J. Heeger

Email: david.heeger@nyu.edu

\section{Table of contents}

Detailed Methods

Input drive: temporal prefilter

Input drive: spatial RFs

Input drive: RF size

Recurrent drive

Normalization weights

Derivations

Notation conventions

Fixed point

Effective gain

Effective time constant

Variants

Bifurcation Analysis

Stochastic resonance

References
1

1

2

3

4

4

5

5

5

7

8

9

14

16

18

\section{Detailed Methods}

Input drive: temporal prefilter

Simulated simple-cell responses $\mathbf{y}$ depended on an input drive $\mathbf{z}$, computed as a weighted sum of LGN inputs $\mathbf{x}$ (Eq. 1). LGN inputs were presumed to have two types of temporal impulse response functions with quadrature phases (Fig. S1a), and each of the two temporal responses were paired with each of the various spatial receptive fields (RFs).

The temporal prefilter was a recursive quadrature temporal filter, that itself was based on ORGaNICs (1). For real-valued inputs:

$$
\begin{aligned}
& \tau_{x} \frac{d x}{d t}=-x+\lambda\left(x_{0}+\operatorname{Im}(x)\right)+(1-\lambda) \hat{x} \\
& \hat{x}=w x \\
& w=1+i 2 \pi \tau \omega
\end{aligned}
$$


where $x_{0}(t)$ is the input and $x(t)$ is the filter output. The value of $\lambda$ determines the effective time constant, and the value of $\omega$ determines the preferred temporal frequency. For complex-valued inputs:

$$
\tau_{x} \frac{d x}{d t}=-x+\lambda x_{0}+(1-\lambda) \hat{x}
$$

The temporal filter was cascaded, analogous to cascading a standard exponential lowpass filter. The response of the $n^{\text {th }}$ filter in the cascade was:

$$
\begin{aligned}
& \tau_{x} \frac{d x_{n}}{d t}=-x_{n}+\lambda x_{n-1}+(1-\lambda) \hat{x}_{n}, \\
& \hat{x}_{n}=w x_{n}, \\
& w=1+i 2 \pi \tau_{x} \omega
\end{aligned}
$$

for $n=1$ to $N$ (i.e., $x_{N}$ corresponds to the LGN responses). The response of the first filter in the cascade was:

$$
\tau_{x} \frac{d x_{1}}{d t}=-x_{1}+\lambda\left(x_{0}+\operatorname{Im}\left(x_{1}\right)\right)+(1-\lambda) \hat{x}_{1}
$$

where $x_{0}(t)$ was the input stimulus. The parameter values for the prefilters were: $N=2 ; \lambda=0.04 ; \tau_{x}=1 \mathrm{~ms}$; $\omega=8 \mathrm{~Hz}$ (i.e., matching the preferred temporal frequency of the simulated cortical neurons).

\section{Input drive: spatial RFs}

Simulated simple-cell responses depended on a weighted sum of the LGN inputs. The rows of the encoding matrix $\mathbf{W}_{z x}$ in Eq. 1 were the spatial RFs of the simple-cells (Figs. S1b,c,d); $\mathbf{W}_{z x}$ was an $N x M$ matrix of weights where $N$ is the number of simple-cells and $M$ is the number of LGN inputs. The LGN inputs comprise pairs of ON- and OFF-center RFs (Fig. 2, center-surround weights), each halfwave rectified, and the input drive comprises differences between each such pair of LGN inputs (Fig. 2, solid and dashed blue lines), so that the input drive is a linear sum of the underlying (unrectified) LGN responses.

Spatial filters were based on the steerable pyramid, a subband image transform that decomposes an image into orientation and spatial frequency (SF) channels (2). The steerable pyramid simulated the responses of a large number of linear RFs, each of which computed a weighted sum of the stimulus image; the weights determined the orientation and SF tuning. There were 12 orientation tuning curves (Fig. S1b). The RFs were defined so that they covered all orientations, SFs, and spatial locations evenly, i.e., the sum of the squares was exactly equal to one (Fig. S1b,c). For each SF and orientation, there were 4 spatial phases and 2 temporal prefilters. For each orientation and SF, there were RFs with four different phases, like odd- and even-phase Gabor filters along with their anti-phase complements. Each of these 4 spatial phases were combined with each of the two temporal prefilters, yielding simplecells with 4 temporal phases. The responses of these space-time separable linear filters provided the input drives to the population of simple-cells. The end result was that $\mathrm{V} 1$ simple-cell responses behaved like spatiotemporal linear filters with various spatial RF locations, orientation preferences, and different temporal phases, that were half-squared (halfwave rectified and squared) (3) and normalized. The responses of a second population of direction-selective simple-cells may be computed as a weighted sum of these space-time separable simple-cells (4).

V1 complex-cell responses were simulated by summing the different temporal phases of the simplecell responses. Because the response of each simple-cell was half-squared, the sum computed what has been called an "energy" response $(3,4)$. The energy response depended on the local spectral energy within a spatial region of the stimulus, for a particular orientation and SF. Because the simple-cell responses were normalized, the complex-cells behaved like normalized, spatiotemporal energy filters.

Because the full set of SF and orientation channels was expensive to compute, the simulation results were instead computed using a reduced set of RFs. For Figs. 3-4, we simulated a collection of neurons with 12 different orientation orientation preferences, but all with the same SF preference and 


\section{Figure S1. Temporal prefilters, receptive fields, and suppressive fields. a. Temporal pre- filters. Blue and orange curves, recursive quad- rature temporal filters. Black curve, amplitude (square-root of sum of squares) of the quadrature pair. b. Orientation tuning curves. Different colors correspond to different orientation preferences. Black curve, sum of squares of tuning curves. c. Receptive fields. Different colors correspond to different RF centers. Black curve, sum of squares of RFs. d. Receptive field size increases with ec- centricity. e. Suppressive fields. Different curves correspond to different receptive fields. Each suppressive field (except those near $\pm 60^{\circ}$ eccen- tricity at the edge of the field of view) is about $4 x$ larger than the corresponding receptive field.}

the same RF center. For Figs. 5-6, we simulated a collection of neurons with the same 12 orientation tuning curves, covering the horizontal meridian of the visual field from $-60^{\circ}$ to $60^{\circ}$ eccentricity.

The orientation tuning curves for the RFs in the steerable pyramid are each one cycle of a raised cosine:

$$
\begin{aligned}
& \psi_{j}(\theta) \propto \frac{1}{2}\left(\cos \left(\frac{\pi}{60}\left(\theta-\theta_{j}\right)\right)+1\right) \\
& \text { for }-\pi \leq \frac{\pi}{60}\left(\theta-\theta_{j}\right) \leq \pi, \text { and } \psi_{j}(\theta)=0 \text { otherwise, }
\end{aligned}
$$
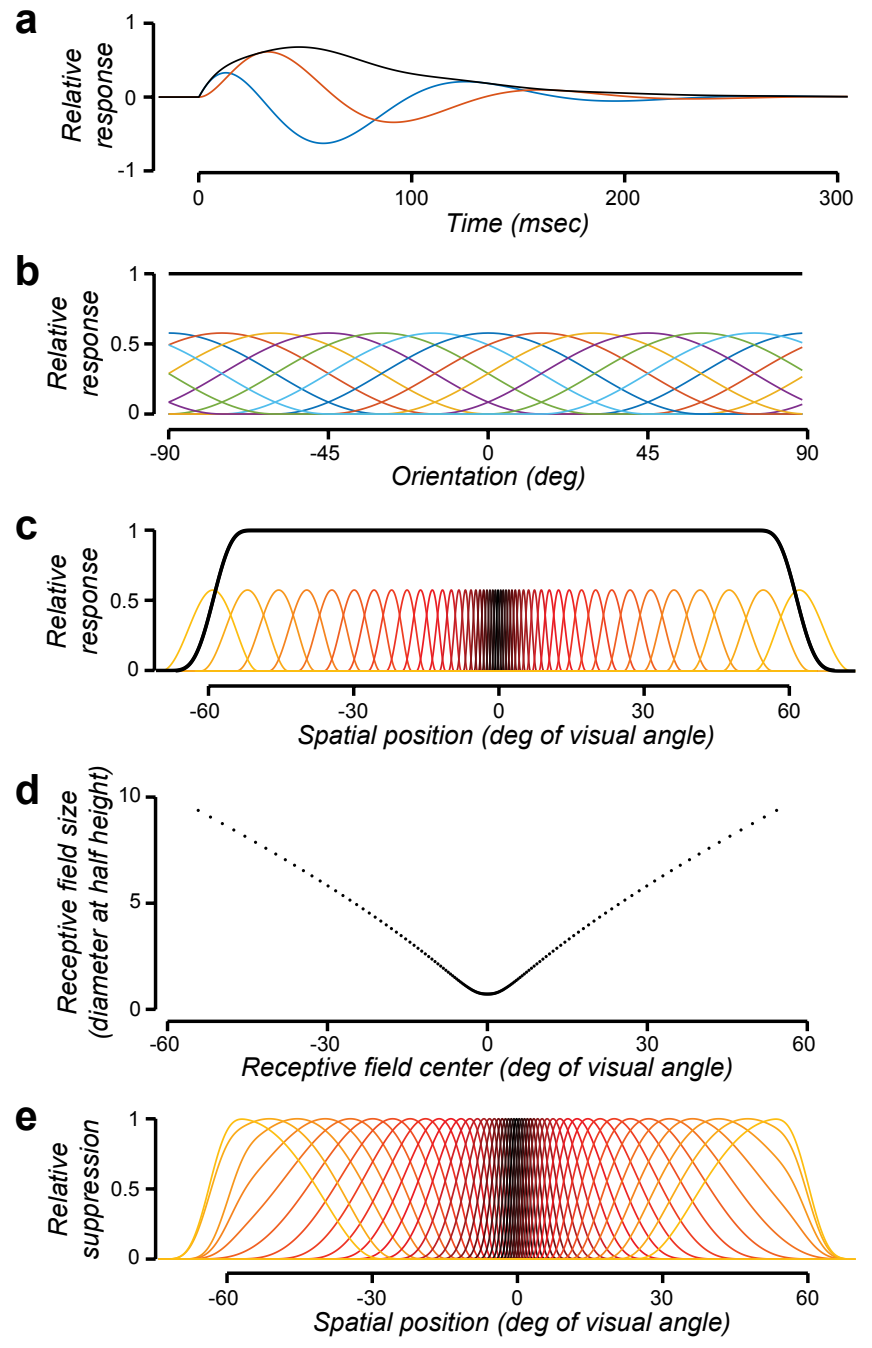

(S5)

where $\theta$ is stimulus orientation (in units of degrees), $\theta_{j}$ is the preferred preferred orientation of the $j^{\text {th }}$ neuron, $\psi_{j}$ is the tuning curve, and the proportionality constant was chosen such that the sum of the squares of the tuning curves was equal to 1 . This corresponds to an orientation bandwidth of $22^{\circ}$ (half width at half height), given that firing rate responses were half-squared (Eq. 2).

\section{Input drive: RF size}

The spatial RFs covered the visual field from $-60^{\circ}$ to $60^{\circ}$ eccentricity, and tiled the visual field so that the sum of the squares of the RFs was equal to 1 (Fig. S1c). RF size increased with eccentricity (Figs. S1d), approximating measurements of V1 RF size and cortical magnification (5-9).

RF size increased with eccentricity (Fig. S1d), approximating measurements of V1 RF size and cortical magnification (5-9). Specifically, we warped visual space:

$$
\xi^{\prime}=\lambda|\xi|^{3.5}+(1-\lambda) \xi
$$

where $0<\xi^{\prime}<1$ is the eccentricity in the visual field after warping and where $0<\xi<1$ is the eccentricity before warping. Both $\xi$ and $\xi^{\prime}$ are each unit-less quantities, expressed as a proportion of the field of view. To convert to eccentricity in units of degrees of visual angle, we multiplied by the field of view, e.g., $60 \xi$ was eccentricity in units of degrees of visual angle when the field of view was $\pm 60^{\circ}$ eccentricity. 
bioRxiv preprint doi: https://doi.org/10.1101/2020.03.22.002634; this version posted May 30, 2020. The copyright holder for this preprint (which was not certified by peer review) is the author/funder. All rights reserved. No reuse allowed without permission.

Dynamic Normalization SI Appendix

May 30, 2020

RFs size was uniform in the unwarped space, with raised cosine profiles (similar to Eq. S5) so as to cover all spatial locations evenly. RF sizes were warped along with the warping of visual space

(Fig. S1c,d).

\section{$\underline{\text { Recurrent drive }}$}

Simulated simple-cell responses $\mathbf{y}$ also depended on a recurrent drive $\hat{\mathbf{y}}$, computed as a weighted sum of the square-root of the responses $\mathbf{y}$. The recurrent weight matrix $\mathbf{W}_{\hat{y} y}$ was an $N \mathrm{x} N$ matrix. An example of a simple-cell's recurrent drive equals the difference between the square root of its own firing rate and the square root of the response of another simple-cell with a complementary RF, i.e., with opposite ON- and OFF- subregions (Fig. 2, solid and dashed green lines). This difference reconstructs the underlying (unrectified) membrane potential fluctuations $\hat{y}_{j}=v_{j}$, such that the input drive $z_{j}$ is lowpass filtered by Eq. 1 to yield the membrane potential $v_{j}$. The effective time constant of the lowpass filter depends on the intrinsic time constant $\tau_{v}$ and the modulator responses $a_{j}$.

We used an alternative recurrent weight matrix that combined the responses of simple-cells with all 4 temporal phases (anti-phase and quadrature phase). The recurrent drive acted as a bandpass filter on the input drive, with any desired preferred temporal frequency $\omega$, and with a bandwidth that depends on the effective time constant of the circuit. Specifically:

$$
\begin{aligned}
& \hat{y}_{j .0}=\left(\sqrt{y_{j, 0}}-\sqrt{y_{j, 180}}\right)-2 \pi \omega \tau_{v}\left(\sqrt{y_{j, 90}}-\sqrt{y_{j, 270}}\right), \\
& \hat{y}_{j .180}=\left(\sqrt{y_{j, 180}}-\sqrt{y_{j, 0}}\right)-2 \pi \omega \tau_{v}\left(\sqrt{y_{j, 270}}-\sqrt{y_{j, 90}}\right), \\
& \hat{y}_{j .90}=2 \pi \omega \tau_{v}\left(\sqrt{y_{j, 0}}-\sqrt{y_{j, 180}}\right)+\left(\sqrt{y_{j, 90}}-\sqrt{y_{j, 270}}\right), \\
& \hat{y}_{j .270}=2 \pi \omega \tau_{v}\left(\sqrt{y_{j, 180}}-\sqrt{y_{j, 0}}\right)+\left(\sqrt{y_{j, 270}}-\sqrt{y_{j, 90}}\right) .
\end{aligned}
$$

The subscript $\varphi$ in $\hat{y}_{j, \varphi}$ is the temporal phase of the simple-cell responses. Simple-cells with $90^{\circ}$ (quadrature phase) and $180^{\circ}$ (anti-phase) phase relationships are adjacent to one another in V1 and the antiphase pairs exhibit strong mutual inhibition (10).

The recurrent drive $\hat{y}_{j}$ is a prediction over time of the principal cell responses $(11,12)$. Information processing in the brain is dynamic; dynamic and predictive processing is needed to control behavior in sync with or in advance of changes in the environment. Without prediction, behavioral responses to environmental events will always be too late because of the lag or latency in sensory and motor processing. Prediction is a key component of theories of motor control and in explanations of how an organism discounts sensory input caused by its own behavior (e.g., 13, 14, 15). Prediction has also been hypothesized to be essential in sensory and perceptual processing (e.g., 16, 17, 18). A further generalization of Eq. S7 computes a weighted sum of neural responses with different preferred temporal frequencies $\omega$ to better predict each neuron's response over time.

\section{Normalization weights}

The normalization pool included all orientations (evenly weighted) at the center of a neuron's RF, and included only orientations near the preferred orientation at spatial locations surrounding the RF. The spatial size of the normalization pool was about 4x larger than the RF (Fig. S1e), except where it was limited in size near the edge of the field of view (near $\pm 60^{\circ}$ eccentricity). The normalization weight matrix $\mathbf{W}$ was scaled such that the effective gain was $g=1$ for a full-contrast, full-field grating with a neuron's preferred spatiotemporal frequency and orientation. 


\section{Derivations}

\section{Notation conventions}

$\mathbf{x}^{2}$ : element-by-element squaring

$\sqrt{\mathbf{x}}$ : element-by-element positive square root

$\frac{\mathbf{x}}{\mathbf{y}}$ : element-by-element division

$\mathbf{y}$

$D(\mathbf{x})$ : diagonal matrix with $\mathbf{x}$ along diagonal

1: vector of 1 's

$$
\begin{aligned}
& y^{+}=\lfloor x\rfloor^{2}=(\max (x, 0))^{2} \\
& y^{-}=\lfloor-x\rfloor^{2}=(\max (-x, 0))^{2}
\end{aligned}
$$

\section{Fixed point}

To derive the fixed point (Eq. 7) of the dynamical system given by Eqs. 1-6:

$$
\begin{aligned}
& \tau_{v} \frac{d v_{j}}{d t}=-v_{j}+\left(\frac{b_{0}}{1+b_{0}}\right) z_{j}+\left(\frac{1}{1+a_{j}}\right)\left(\sqrt{y_{j}^{+}}-\sqrt{y_{j}^{-}}\right), \\
& \tau_{a} \frac{d a_{j}}{d t}=-a_{j}+\sqrt{u_{j}}+a_{j} \sqrt{u_{j}}, \\
& \tau_{u} \frac{d u_{j}}{d t}=-u_{j}+\sum_{k} w_{j k}\left(y_{k}^{+}+y_{k}^{-}\right) u_{k}+\left(\frac{\sigma b_{0}}{1+b_{0}}\right)^{2}, \\
& y_{j}^{+}=\left\lfloor v_{j}\right\rfloor^{2} \text { and } y_{j}^{-}=\left\lfloor-v_{j}\right\rfloor^{2}, \\
& \text { i.e., } v_{j}=\left(\sqrt{y_{j}^{+}}-\sqrt{y_{j}^{-}}\right) \text {and } v_{j}^{2}=\left(y_{j}^{+}+y_{j}^{-}\right),
\end{aligned}
$$

$\mathrm{b}_{0}>0$ is a constant,

$\sigma>0$ is a constant,

$w_{j k}>0$ are the elements of the normalization weight matrix $\mathbf{W}$,

the values of $u_{j}$ are subject to the constraint that $u_{j} \geq\left(\frac{\sigma b_{0}}{1+b_{0}}\right)^{2}>0$.

Here, we have imposed three additional assumptions: 1) The constraint on $u_{j}$ may be interpreted to mean that this population of neurons has a small spontaneous firing rate, even when the membrane potential is hyperpolarized. 2) The recurrent drive equals the difference between the square root of its own firing rate and the square root of the response of another simple-cell with a complementary RF (i.e., with opposite ON- and OFF- subregions). 3) The normalization weights are identical for contributions to the normalization pool from complementary RFs. 
Set the derivatives in Eq. S8 equal to 0 and simplify:

$$
\begin{aligned}
& \frac{d \mathbf{v}}{d t}=0: \\
& v_{j}=\left(\frac{b_{0}}{1+b_{0}}\right) z_{j}+\left(\frac{1}{1+a_{j}}\right)\left(\sqrt{y_{j}^{+}}-\sqrt{y_{j}^{-}}\right), \\
& v_{j}=\left(\frac{b_{0}}{1+b_{0}}\right) z_{j}+\left(\frac{1}{1+a_{j}}\right) v_{j}, \\
& \left(\frac{a_{j}}{1+a_{j}}\right) v_{j}=\left(\frac{b_{0}}{1+b_{0}}\right) z_{j}, \\
& \left(\frac{a_{j}}{1+a_{j}}\right)^{2} v_{j}^{2}=\left(\frac{b_{0}}{1+b_{0}}\right)^{2} z_{j}^{2}, \\
& D\left(\mathbf{v}^{2}\right)\left(\frac{\mathbf{a}}{1+\mathbf{a}}\right)^{2}=\left(\frac{b_{0}}{1+b_{0}}\right)^{2} \mathbf{z}^{2} .
\end{aligned}
$$

$$
\begin{aligned}
& \frac{d \mathbf{u}}{d t}=0: \\
& u_{j}=\sum_{k} w_{j k}\left(y_{k}^{+}+y_{k}^{-}\right) u_{k}+\left(\frac{\sigma b_{0}}{1+b_{0}}\right)^{2} \\
& u_{j}=\sum_{k} w_{j k} v_{k}^{2} u_{k}+\left(\frac{\sigma b_{0}}{1+b_{0}}\right)^{2} \\
& \mathbf{u}=\mathbf{W} D\left(\mathbf{v}^{2}\right) \mathbf{u}+\left(\frac{\sigma b_{0}}{1+b_{0}}\right)^{2} \\
& \left(\mathbf{I}-\mathbf{W} D\left(\mathbf{v}^{2}\right)\right) \mathbf{u}=\left(\frac{\sigma b_{0}}{1+b_{0}}\right)^{2} \mathbf{1} \\
& \mathbf{u}=\left(\frac{\sigma b_{0}}{1+b_{0}}\right)^{2}\left(\mathbf{I}-\mathbf{W} D\left(\mathbf{v}^{2}\right)\right)^{-1} \mathbf{1}
\end{aligned}
$$

$$
\begin{aligned}
& \frac{d \mathbf{a}}{d t}=0: \\
& a_{j}=\sqrt{u_{j}}+a_{j} \sqrt{u_{j}}, \\
& a_{j}=\left(1+a_{j}\right) \sqrt{u_{j}}, \\
& \left(\frac{a_{j}}{1+a_{j}}\right)^{2}=u_{j}, \\
& \left(\frac{\mathbf{a}}{1+\mathbf{a}}\right)^{2}=\mathbf{u}
\end{aligned}
$$


Dynamic Normalization SI Appendix

May 30, 2020

Combine the last line of Eq. S10 with the last line of Eq. S11:

$$
\begin{aligned}
& \left(\frac{\mathbf{a}}{1+\mathbf{a}}\right)^{2}=\left(\frac{\sigma b_{0}}{1+b_{0}}\right)^{2}\left(\mathbf{I}-\mathbf{W} D\left(\mathbf{v}^{2}\right)\right)^{-1} \mathbf{1}, \\
& \left(\mathbf{I}-\mathbf{W} D\left(\mathbf{v}^{2}\right)\right)\left(\frac{\mathbf{a}}{1+\mathbf{a}}\right)^{2}=\left(\frac{\sigma b_{0}}{1+b_{0}}\right)^{2} \mathbf{1}, \\
& \left(\mathbf{I}-\mathbf{W} D\left(\mathbf{v}^{2}\right)\right)\left(\frac{\mathbf{a}}{1+\mathbf{a}}\right)^{2}=\left(\frac{\sigma b_{0}}{1+b_{0}}\right)^{2} D\left(\mathbf{v}^{2}\right)^{-1} \mathbf{v}^{2}, \\
& D\left(\mathbf{v}^{2}\right)\left(\mathbf{I}-\mathbf{W} D\left(\mathbf{v}^{2}\right)\right)\left(\frac{\mathbf{a}}{1+\mathbf{a}}\right)^{2}=\left(\frac{\sigma b_{0}}{1+b_{0}}\right)^{2} \mathbf{v}^{2}, \\
& {\left[D\left(\mathbf{v}^{2}\right)-D\left(\mathbf{v}^{2}\right) \mathbf{W} D\left(\mathbf{v}^{2}\right)\right]\left(\frac{\mathbf{a}}{1+\mathbf{a}}\right)^{2}=\left(\frac{\sigma b_{0}}{1+b_{0}}\right)^{2} \mathbf{v}^{2},} \\
& \left(\mathbf{I}-D\left(\mathbf{v}^{2}\right) \mathbf{W}\right) D\left(\mathbf{v}^{2}\right)\left(\frac{\mathbf{a}}{1+\mathbf{a}}\right)^{2}=\left(\frac{\sigma b_{0}}{1+b_{0}}\right)^{2} \mathbf{v}^{2} .
\end{aligned}
$$

Substitute from the last line of Eq. $\mathbf{S 9}$ and simplify:

$$
\begin{aligned}
& \left(\frac{\sigma b_{0}}{1+b_{0}}\right)^{2} \mathbf{v}^{2}=\left(\mathbf{I}-D\left(\mathbf{v}^{2}\right) \mathbf{W}\right)\left(\frac{b_{0}}{1+b_{0}}\right)^{2} \mathbf{z}^{2}, \\
& \sigma^{2} \mathbf{v}^{2}=\left(\mathbf{I}-D\left(\mathbf{v}^{2}\right) \mathbf{W}\right) \mathbf{z}^{2}, \\
& \sigma^{2} \mathbf{v}^{2}=\mathbf{z}^{2}-D\left(\mathbf{v}^{2}\right) \mathbf{W} \mathbf{z}^{2}, \\
& \sigma^{2} \mathbf{v}^{2}=\mathbf{z}^{2}-D\left(\mathbf{W} \mathbf{z}^{2}\right) \mathbf{v}^{2}, \\
& \sigma^{2} \mathbf{v}^{2}+D\left(\mathbf{W} \mathbf{z}^{2}\right) \mathbf{v}^{2}=\mathbf{z}^{2}, \\
& \left(\sigma^{2} \mathbf{I}+D\left(\mathbf{W} \mathbf{z}^{2}\right)\right) \mathbf{v}^{2}=\mathbf{z}^{2}, \\
& D\left(\sigma^{2}+\mathbf{W} \mathbf{z}^{2}\right) \mathbf{v}^{2}=\mathbf{z}^{2}, \\
& \mathbf{v}^{2}=\left(D\left(\sigma^{2}+\mathbf{W} \mathbf{z}^{2}\right)\right)^{-1} \mathbf{z}^{2}, \\
& \mathbf{v}^{2}=\frac{\mathbf{z}}{\sigma^{2}+\mathbf{W} \mathbf{z}^{2}}, \\
& \mathbf{y}^{+}=\frac{\lfloor\mathbf{z}\rfloor^{2}}{\sigma^{2}+\mathbf{W} \mathbf{z}^{2}} \text { and } \mathbf{y}^{-}=\frac{\lfloor-\mathbf{z}\rfloor^{2}}{\sigma^{2}+\mathbf{W} \mathbf{z}^{2}} .
\end{aligned}
$$

\section{Effective gain}

To derive the an expression for the effective gain, we begin with the last line of Eq. S13. The effective gain is the ratio of each element of $\mathbf{y}$ to each element of $\mathbf{z}^{2}$ :

$$
\mathbf{g}=\frac{1}{\sigma^{2}+\mathbf{W z}^{2}}
$$

Because the normalization weights $\mathbf{W}$ are all positive:

$$
\mathbf{g}<g_{\max } \text { where } g_{\max }=\frac{1}{\sigma^{2}} .
$$

The expression for the effective gain (Eq. S14) simplifies for stimuli comprised of sinusoidal gratings, because the normalization weight matrix $\mathbf{W}$ was scaled such that the effective gain $g=1$ for a full-contrast, full-field grating with a neuron's preferred spatiotemporal frequency and orientation. For a single sinusoidal grating $\mathbf{W z}^{2}$ is proportional to the squared contrast $c^{2}$. For sums of gratings, $\mathbf{W} \mathbf{z}^{2}$ is proportional to a weighted sum of the squared contrasts (e.g., as expressed by Eq. 9 for a test grating of pre- 
ferred orientation restricted to the RF and a mask grating that by itself does not evoke a response).

\section{Effective time constant}

To derive an expression for effective time constant, we begin by rewriting the first line of Eq. S8:

$$
\begin{aligned}
& \tau_{v} \frac{d v_{j}}{d t}=-v_{j}+\left(\frac{b_{0}}{1+b_{0}}\right) z_{j}+\left(\frac{1}{1+a_{j}}\right) v_{j}, \\
& \tau_{v} \frac{d v_{j}}{d t}=-\left(\frac{a_{j}}{1+a_{j}}\right) v_{j}+\left(\frac{b_{0}}{1+b_{0}}\right) z_{j}, \\
& \tau_{j} \frac{d v_{j}}{d t}=-v_{j}+\left(\frac{b_{0}}{1+b_{0}}\right)\left(\frac{1+a_{j}}{a_{j}}\right) z_{j}, \\
& \text { where } \tau_{j}=\left(\frac{1+a_{j}}{a_{j}}\right) \tau_{v} .
\end{aligned}
$$

Substitute from the last line of Eq. S11:

$$
\tau=\frac{\tau_{v}}{\sqrt{\mathbf{u}}}
$$

To express the effective time constant in terms of the effective gain, we first derive another expression for the effective gain by combining the fixed point from Eq. S9 with the last line of Eq. S11:

$$
\begin{aligned}
& \mathbf{y}=\left(\frac{1+\mathbf{a}}{\mathbf{a}}\right)^{2}\left(\frac{b_{0}}{1+b_{0}}\right)^{2} \mathbf{z}^{2}, \\
& \mathbf{g}=\left(\frac{1+\mathbf{a}}{\mathbf{a}}\right)^{2}\left(\frac{b_{0}}{1+b_{0}}\right)^{2}, \\
& \mathbf{g}=\left(\frac{1}{\mathbf{u}}\right)\left(\frac{b_{0}}{1+b_{0}}\right)^{2} .
\end{aligned}
$$

I.e.,

$$
\frac{1}{\sqrt{\mathbf{u}}}=\left(\frac{1+b_{0}}{b_{0}}\right) \sqrt{\mathbf{g}} .
$$

Combine Eqs. S17 and S19:

$$
\tau=\tau_{v}\left(\frac{1+b_{0}}{b_{0}}\right) \sqrt{\mathbf{g}}
$$




\section{Variants}

The circuit model expressed by Eqs. 1-6 is but one example of a family of dynamical systems models of normalization, each of which implements normalization via recurrent amplification.

A simple variant of an ORGaNIC normalization circuit is expressed by the following coupled pair of neural integrators:

$$
\begin{gathered}
\tau_{y} \frac{d y_{j}}{d t}=-y_{j}+b_{0} z_{j}+\left(1-a_{j}\right) y_{j}, \\
\tau_{a} \frac{d a_{j}}{d t}=-a_{j}+\sum_{k} w_{j k} y_{k} a_{k}+\sigma b_{0} .
\end{gathered}
$$

To derive the fixed point for this dynamical system, set the derivatives equal to 0 and simplify:

$$
\begin{aligned}
& \frac{d y_{j}}{d t}=0: a_{j} y_{j}=b_{0} z_{j}, \\
& \frac{d a_{j}}{d t}=0: a_{j}=\sum_{k} w_{j k} y_{k} a_{k}+\sigma b_{0} .
\end{aligned}
$$

Substitute the first line of Eq. $\mathbf{S 2 2}$ into the second line of Eq. S22:

$$
a_{j}=b_{0}\left(\sum_{k} w_{j k} z_{k}+\sigma\right) \text {. }
$$

Substitute this back into the first line of Eq. S22:

$$
\begin{aligned}
& b_{0}\left(\sum_{k} w_{j k} z_{k}+\sigma\right) y_{j}=b_{0} z_{j}, \\
& \left(\sum_{k} w_{j k} z_{k}+\sigma\right) y_{j}=z_{j}, \\
& y_{j}=\frac{z_{j}}{\sigma+\sum_{k} w_{j k} z_{k}} .
\end{aligned}
$$

There is, however, considerable empirical evidence that the firing rate responses of $\mathrm{V} 1$ neurons depend on the contrast energy of the stimulus, i.e., the square of the input drive $(3,19,20)$. In addition, squaring offers theoretical advantages. First, responses depended on the local spectral energy (ignoring phase) in a local spatiotemporal window of the stimulus $(3,4)$. Second, the summed responses across the population of neurons tile all orientations, SFs, and spatial locations evenly (2), i.e., the sum of the squares is exactly equal to one (Fig. S1b,c).

Elaborating Eq. $\mathbf{S 2 1}$ with halfwave rectification and squaring yields another variant:

$$
\begin{aligned}
& \tau_{v} \frac{d v_{j}}{d t}=-v_{j}+b_{0} z_{j}+\left(1-\sqrt{a_{j}}\right)\left(\sqrt{y_{j}^{+}}-\sqrt{y_{j}^{-}}\right), \\
& \tau_{a} \frac{d a_{j}}{d t}=-a_{j}+\sum_{k} w_{j k} y_{k} a_{k}+\sigma^{2} b_{0}^{2}, \\
& y_{j}=\left\lfloor v_{j}\right\rfloor^{2} .
\end{aligned}
$$

This variant has the same fixed point as Eqs. 1-6 (equivalently Eq. S8); the derivation of the fixed point for this system is very similar to that shown above for Eq. S8. This circuit exhibits different dynamics 
from Eqs. 1-6 even though they both share the same fixed point (see below, Bifurcation Analysis).

Another variant is:

$$
\begin{aligned}
& \tau_{v} \frac{d v_{j}}{d t}=-v_{j}+\left(\frac{b_{0}}{1+b_{0}}\right) z_{j}+\left(\frac{2 a_{j}}{1+a_{j}}\right)\left(\sqrt{y_{j}^{+}}-\sqrt{y_{j}^{-}}\right), \\
& \tau_{a} \frac{d a_{j}}{d t}=-a_{j}-\sqrt{u_{j}}-a_{j} \sqrt{u_{j}}+d, \\
& \tau_{u} \frac{d u_{j}}{d t}=-u_{j}+\sum_{k} w_{j k} y_{k} u_{k}+\left(\frac{\sigma b_{0}}{1+b_{0}}\right)^{2}, \\
& y_{j}=\left\lfloor v_{j}\right\rfloor^{2},
\end{aligned}
$$

where $d>0$. In this variant, we replaced $1 /(1+\mathbf{a})$ in Eq. 1 with $2 \mathbf{a} /(1+\mathbf{a})$, in which $0<\mathbf{a}<1$, with concomitant changes to the equation for a (Eq. 5). When the value of $d=1$, the fixed point for this system is the same as that for Eqs. 1-6 (the derivation follows that shown above for Eqs. 1-6). The dynamics are also similar (the two systems are related by a simple change of variables). But when $d<1$, the gain of this system is reduced.

To derive the gain as a function of $d$, consider the reduced system in which each of the variables is a scalar instead of a vector (i.e., there's only one neuron of each type instead of population of neurons with different RFs, SF preferences, and orientation preferences):

$$
\begin{aligned}
& \tau_{v} \frac{d v}{d t}=-v+\left(\frac{b_{0}}{1+b_{0}}\right) z+\left(\frac{2 a}{1+a}\right) \sqrt{y}, \\
& \tau_{a} \frac{d a}{d t}=-a-\sqrt{u}-a \sqrt{u}+d, \\
& \tau_{u} \frac{d u}{d t}=-u+y u+\left(\frac{\sigma b_{0}}{1+b_{0}}\right)^{2}, \\
& y=v^{2}, \\
& z \geq 0 .
\end{aligned}
$$

The fixed point for this system is derived, again, by setting the derivatives equal to zero:

$$
\begin{aligned}
& \frac{d v}{d t}=0: \\
& v=\left(\frac{b_{0}}{1+b_{0}}\right) z+\left(\frac{2 a}{1+a}\right) v, \\
& \left(\frac{1-a}{1+a}\right) v=\left(\frac{b_{0}}{1+b_{0}}\right) z, \\
& \left(\frac{1-a}{1+a}\right)^{2} y=\left(\frac{b_{0}}{1+b_{0}}\right)^{2} z^{2}, \\
& y=\left(\frac{1+a}{1-a}\right)^{2}\left(\frac{b_{0}}{1+b_{0}}\right)^{2} z^{2} .
\end{aligned}
$$




$$
\begin{aligned}
& \frac{d u}{d t}=0: \\
& u=y u+\left(\frac{\sigma b_{0}}{1+b_{0}}\right)^{2}, \\
& u(1-y)=\left(\frac{\sigma b_{0}}{1+b_{0}}\right)^{2}, \\
& u=\left(\frac{\sigma b_{0}}{1+b_{0}}\right)^{2} \frac{1}{(1-y)} . \\
& \frac{d a}{d t}=0: \\
& (d-a)=\sqrt{u}+a \sqrt{u}, \\
& (d-a)=(1+a) \sqrt{u}, \\
& u=\left(\frac{d-a}{1+a}\right)^{2} .
\end{aligned}
$$

Combine the last line of Eq. S29 with the last line of Eq. S30:

$$
\left(\frac{1+a}{d-a}\right)^{2}=\left(\frac{1+b_{0}}{\sigma b_{0}}\right)^{2}(1-y) \text {. }
$$

Combine the last line of Eq. S28 with Eq. S31:

$$
\begin{aligned}
& y=\left(\frac{1+a}{1-a}\right)^{2}\left(\frac{b_{0}}{1+b_{0}}\right)^{2} z^{2}, \\
& y=\left(\frac{d-a}{1-a}\right)^{2}\left(\frac{1+a}{d-a}\right)^{2}\left(\frac{b_{0}}{1+b_{0}}\right)^{2} z^{2}, \\
& y=\left(\frac{d-a}{1-a}\right)^{2}(1-y)\left(\frac{1+b_{0}}{\sigma b_{0}}\right)^{2}\left(\frac{b_{0}}{1+b_{0}}\right)^{2} z^{2}, \\
& \sigma^{2} y=\left(\frac{d-a}{1-a}\right)^{2}(1-y) z^{2} .
\end{aligned}
$$

Simplify:

$$
\begin{aligned}
& \sigma^{2} y=\left(\frac{d-a}{1-a}\right)^{2}\left(z^{2}-z^{2} y\right), \\
& y\left(\sigma^{2}+\left(\frac{d-a}{1-a}\right)^{2} z^{2}\right)=\left(\frac{d-a}{1-a}\right)^{2} z^{2}, \\
& y=\frac{\left(\frac{d-a}{1-a}\right)^{2} z^{2}}{\sigma^{2}+\left(\frac{d-a}{1-a}\right)^{2} z^{2}}, \\
& y=\frac{z^{2}}{\left(\sigma^{\prime}\right)^{2}+z^{2}}, \\
& \text { where }\left(\sigma^{\prime}\right)=\left(\frac{1-a}{d-a}\right) \sigma .
\end{aligned}
$$

For $\mathrm{d}<1, \sigma^{\prime}>\sigma$ and the effective gain is reduced.

Some of the other variants are as follows. Each is written as a reduced system in which each of the variables is a scalar instead of a vector, although each can also be expressed as a full population with 

arbitrary (non-negative normalization) weights.

$$
\begin{aligned}
& \tau_{y} \frac{d y}{d t}=-y+b_{0} z+(1-a) y \\
& \tau_{a} \frac{d a}{d t}=-a+y a+\sigma b_{0} \\
& z \geq 0
\end{aligned}
$$

$\tau_{v} \frac{d v}{d t}=-v+b_{0} z+(1-\sqrt{a}) \sqrt{y}$,

$\tau_{a} \frac{d a}{d t}=-a+y a+\sigma^{2} b_{0}^{2}$,

$y=v^{2}$,

$z \geq 0$.

$\tau_{v} \frac{d v}{d t}=-v+b_{0} z+(1-a) \sqrt{y}$,

$\tau_{a} \frac{d a}{d t}=-a+\sqrt{u}$,

$\tau_{u} \frac{d u}{d t}=-u+y u+\sigma^{2} b_{0}^{2}$,

$y=v^{2}$,

$z \geq 0$.

$\tau_{v} \frac{d v}{d t}=-v+\left(\frac{b_{0}}{1+b_{0}}\right) z+\left(\frac{1}{1+a}\right) \sqrt{y}$

$\tau_{a} \frac{d a}{d t}=-a+\sqrt{u}+a \sqrt{u}$,

$\tau_{u} \frac{d u}{d t}=-u+y u+\left(\frac{\sigma b_{0}}{1+b_{0}}\right)^{2}$,

$y=v^{2}$,

$z \geq 0$. 


$$
\begin{aligned}
& \tau_{v} \frac{d v}{d t}=-v+\left(\frac{1}{1+b_{0}}\right) z+\left(\frac{1}{1+a}\right) \sqrt{y}, \\
& \tau_{a} \frac{d a}{d t}=-a+\sqrt{u}+a \sqrt{u}, \\
& \tau_{u} \frac{d u}{d t}=-u+y u+\left(\frac{\sigma}{1+b_{0}}\right)^{2}, \\
& y=v^{2}, \\
& z \geq 0 . \\
& \tau_{v} \frac{d v}{d t}=-v+\left(\frac{b_{0}}{1+b_{0}}\right) z+\left(\frac{1}{1+a}\right) \sqrt{y}, \\
& \tau_{a} \frac{d a}{d t}=-a+u_{1}+u_{1} a, \\
& \tau_{u_{1}} \frac{d u_{1}}{d t}=-u_{1}+\frac{u_{2}}{u_{1}} \text { such that } u_{1}>0, \\
& \tau_{u_{2}} \frac{d u_{2}}{d t}=-u_{2}+y u_{2}+\left(\frac{\sigma b_{0}}{1+b_{0}}\right)^{2}, \\
& y=v^{2}, \\
& z \geq 0 .
\end{aligned}
$$

Eqs. S35-S39 have the same fixed point. The fixed point for Eq. S34 is similar, but without the squaring. Eqs. S36-S39 exhibit the same or similar dynamics. Eqs. S34-S35 exhibit different dynamics (see below, Bifurcation Analysis). Eq. S34 is the reduced version of Eq. S21, Eq. S35 is the reduced version of Eq. S25, and Eq. S37 is the reduced version of Eqs. 1-6 (equivalently Eq. S8). Eq. S39 eliminates the square root in the expression for $u$; the square root of $y$ may be eliminated analogously. 
bioRxiv preprint doi: https://doi.org/10.1101/2020.03.22.002634; this version posted May 30, 2020. The copyright holder for this preprint (which was not certified by peer review) is the author/funder. All rights reserved. No reuse allowed without permission.

Dynamic Normalization SI Appendix

May 30, 2020

\section{Bifurcation Analysis}

The 2D bifurcation diagrams in Fig. 7e were computed as follows, based on the reduced model (Eq. S37). The fixed points of that system of equations are found by setting all of the derivatives ( $\mathrm{d} v / \mathrm{d} t$, $\mathrm{d} a / \mathrm{d} t$, and $\mathrm{d} u / \mathrm{d} t)$ equal zero:

$$
\begin{aligned}
& v=\frac{z}{\sqrt{\sigma^{2}+z^{2}}}, \\
& a=\frac{\sqrt{u}}{1-\sqrt{u}}, \\
& u=\left(\frac{b_{0}}{1+b_{0}}\right)^{2}\left(\sigma^{2}+z^{2}\right) .
\end{aligned}
$$

There was a different fixed point for each value of the input drive $z$. The Jacobian of Eq. S37 was analyzed to determine if a fixed point is stable (an attractor; Fig. 7a, solid curve) or unstable (typically associated with a limit cycle; Fig. 7a, dashed curve). The Jacobian is a $3 \times 3$ matrix of partial derivatives of Eq. S37 evaluated at the fixed point:

$$
\mathbf{J}=\left(\begin{array}{ccc}
\frac{-1+\sqrt{u}}{\tau_{a}} & 0 & \frac{1+a}{2 \tau_{a} \sqrt{u}} \\
\frac{-v}{\tau_{v}(1+a)^{2}} & \frac{-a}{\tau_{v}(1+a)} & 0 \\
0 & \frac{2 u v}{\tau_{u}} & \frac{v^{2}-1}{\tau_{u}}
\end{array}\right)
$$

To compute the Jacobian, the derivatives must be continuous, which is the case for our system of equations when $a, v$, and $u>0$, which is the regime that we care about. Stability depends on the eigenvalues of this Jacobian matrix. If the real parts of all of the eigenvalues are negative, then the fixed point is stable. If at least one eigenvalue has a positive real part, then the fixed point is unstable because this means that there is at least one direction along which a trajectory will not return back to the fixed point. The intuition is like dropping a marble on a paraboloid. If the paraboloid is concave upward (like a bowl) then the marble will roll back to the fixed point at the bottom of the bowl. If it is concave downward (like an upside down bowl) or a hyperbolic paraboloid (a saddle), then there is at least one direction in which the marble will roll downward away from the fixed point.

A Hopf bifurcation occurs when a spiraling fixed point changes from stable to unstable (or vice versa), i.e., when the real part of a complex-conjugate pair of eigenvalues changes sign. The point at which the real part of the complex-valued eigenvalues is equal to zero is the Hopf bifurcation point. Requiring that the real part of the eigenvalues equal zero is equivalent to requiring a particular set of constraints on the determinant and trace of the Jacobian matrix (21), although we omit writing the exact set of constraints here as they are cumbersome. We found the points that satisfy these constraints by solving a polynomial in our six parameters: $z, \sigma, b_{0}, \tau_{v}, \tau_{a}$, and $\tau_{u}$. Since we have a high dimensional parameter space, finding the roots of this polynomial is itself a hard problem. We therefore restricted our analysis to a subset of the parameter space by keeping $\sigma$ and $b_{0}$ fixed and characterizing 3D slices through the other parameters, either $\left(z, \tau_{v}, \tau_{a}\right)$ or $\left(z, \tau_{a}, \tau_{u}\right)$. The condition on the eigenvalues to change sign alone is not sufficient to guarantee the existence of a limit cycle, although one almost always does arise (22). We therefore checked that a limit cycle did indeed arise (for each fixed point) using AUTO-07p (Version 0.8), a bifurcation analysis software platform for ordinary differential equations (23). AUTO-07p can identify the Hopf Bifurcation points, compute the emergent oscillation period, and track changes in the period as a function of a parameter. For each point on the grid of values for $z$ and $\tau_{u}$, we determined a unique stable periodic solution (limit cycle) and computed its period. Doing so identified the onset of oscillations, i.e., the point of a Hopf bifurcation (Fig. 7e, solid curves circumscribing grayscale shaded regions), and computed the frequencies of the observed oscillations when a limit cycle appeared (Fig. 7e, grayscale).

There are parameter regimes in which the system exhibits bistability such that a limit cycle (stable periodic solution) coexists with a stable fixed point. This is unlike the behavior in Fig. $\mathbf{7}$ for which each parameter set corresponds to a unique attractor. An example of such bistable dynamics corresponds to 
bioRxiv preprint doi: https://doi.org/10.1101/2020.03.22.002634; this version posted May 30, 2020. The copyright holder for this preprint (which was not certified by peer review) is the author/funder. All rights reserved. No reuse allowed without permission.

Dynamic Normalization SI Appendix

May 30, 2020

the parameter set given by $b_{0}=5, \tau_{a}=2$, and $\tau_{u}=1$. For these parameter values, and for increasing values of the input drive $z$, we found a periodic solution (i.e., a limit cycle) extending past the point where the Hopf bifurcation occurs. This means that there are two stable attractors for the same value of the input drive: a stable fixed point and a stable limit cycle, i.e., convergence to both steady state and oscillations. The initial values of $v, a$, and $u$ determine which of the two behaviors will be observed.

We proved that the alternative 2-dimensional circuit, given by Eq. S35, does not exhibit oscillations. The fixed points of that system of equations correspond to where the derivatives $(\mathrm{d} v / \mathrm{d} t$ and $\mathrm{d} a / \mathrm{d} t)$ are equal to zero:

$$
\begin{aligned}
& v=\frac{z}{\sqrt{\sigma^{2}+z^{2}}}, \\
& a=b_{0}^{2}\left(\sigma^{2}+z^{2}\right) .
\end{aligned}
$$

These two equations are continuously differentiable, so we derived an expression for the eigenvalues of the Jacobian at the fixed point. The Jacobian is given by:

$$
\mathbf{J}=\left(\begin{array}{cc}
\frac{-\sqrt{a}}{\tau_{v}} & \frac{-v}{2 \tau_{v} \sqrt{a}} \\
\frac{2 v a}{\tau_{a}} & \frac{v^{2}-1}{\tau_{a}}
\end{array}\right)
$$

The eigenvalues are computed by solving the characteristic equation:

$$
\operatorname{det}(\mathbf{J}-\lambda \mathbf{I})=\left(\frac{\sqrt{a}}{\tau_{v}}-\lambda\right)\left(\frac{v^{2}-1}{\tau_{a}}-\lambda\right)+\frac{v^{2} \sqrt{a}}{\tau_{a} \tau_{v}}
$$

Solving for $\lambda$ gives:

$$
\lambda_{1,2}=\frac{1}{2}\left(\left(\frac{v^{2}-1}{\tau_{a}}-\frac{\sqrt{a}}{\tau_{v}}\right) \pm \sqrt{\left(\frac{v^{2}-1}{\tau_{a}}-\frac{\sqrt{a}}{\tau_{v}}\right)^{2}-\frac{4 \sqrt{a}}{\tau_{v} \tau_{a}}}\right) .
$$

For a hopf bifurcation, the real part of this complex-conjugate pair of eigenvalues must be zero with non-zero imaginary part. However, this would require:

$$
\left(\frac{v^{2}-1}{\tau_{a}}-\frac{\sqrt{a}}{\tau_{v}}\right)=0 \text {. }
$$

But, in fact, the expression in Eq. S46 (same as the first term in Eq. S45) is strictly less than zero because $v<1$, and $\tau_{v}, \tau_{a}$, and $a>0$. So this system will never change its stability. To determine the type of stability that the system exhibits we just need to determine the sign of the real parts of the eigenvalues. The expression under the square root in Eq. S45 is smaller than the first term in Eq. S45, and we have already determined that the first term in Eq. $\mathbf{S 4 5}$ is less than zero. Consequently, the real parts of the eigenvalues are negative, the fixed point is stable, and all of the trajectories approach the fixed point as $t \rightarrow \infty$.

The circuit expressed by Eq. S36 is similar to that expressed by Eq. S35, but with two modulator cells instead of one. The extra modulator cell merely adds a second stage of lowpass filtering. The resulting cascade of two exponential lowpass filters imposes a time delay that suffices for oscillations to emerge. 
bioRxiv preprint doi: https://doi.org/10.1101/2020.03.22.002634; this version posted May 30, 2020. The copyright holder for this preprint (which was not certified by peer review) is the author/funder. All rights reserved. No reuse allowed without permission.

Dynamic Normalization SI Appendix

May 30, 2020

\section{Stochastic resonance}

With noise added to the input, we observed stochastic resonance in the gamma frequency range even for weak inputs (below the bifurcation), and that the noise spectrum is shaped by recurrent normalization to exhibit a resonant peak in the gamma-frequency range (Fig. S2). Specifically, we used the reduced system of Eq. S37 to simulate responses to noisy inputs. The input drive was a step function (constant over time after onset) with Gaussian noise added. The noisy input drive was lowpass filtered using Eq. S3 with $\omega=0 \mathrm{~Hz}$ and $n=2$ before being normalized by Eq. S37.

With noise added, the phases of the oscillatory responses to each of a series of step inputs were synchronized to the onset of the input drive for a period of time following the onset. The response phases then drifted over time and desynchronized (Fig. S2a, different colors correspond to repeated simulations with different noise samples).

a

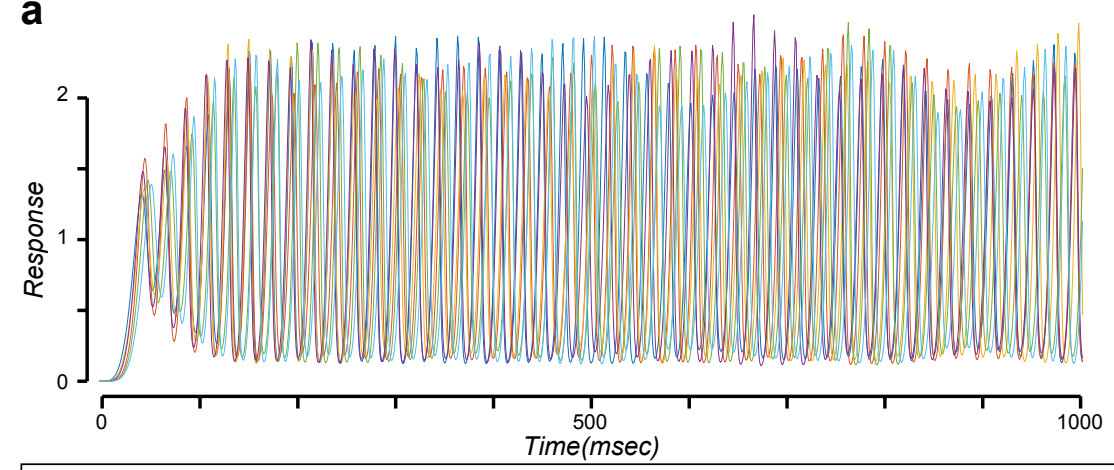

b

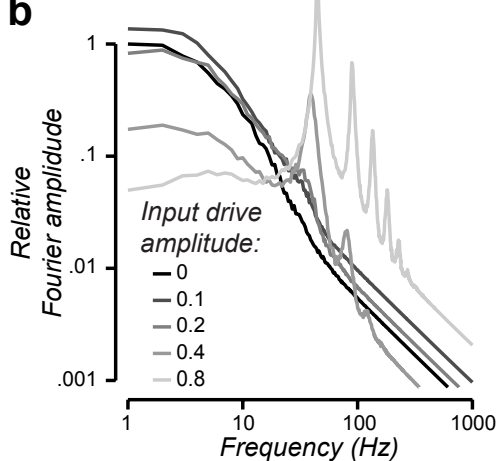

Figure S2. Stochastic resonance. a. Response time series for noisy input drive (input drive amplitude $z=1$ ). Different colors correspond to repeated simulations with different noise samples. The oscillatory responses were induced, not evoked. Specifically, the phases of the oscillatory responses were synchronized immediately after the onset of the input drive, but then drifted over time and desynchronized. b. Fourier amplitude of the responses for each of several input drive amplitudes. Different gray shades correspond to different input drive amplitudes. Increased power in the gamma frequency range is evident input drives as small as $z=0.2$.

The Fourier amplitude of the responses was computed by running the simulation for 100 sec, ignoring the first $1 \mathrm{sec}$ of the responses (to ignore the synchronized part), dividing the remaining response time series into $1 \mathrm{sec}$ intervals, computing the Fourier transform of each $1 \mathrm{sec}$ interval, and averaging them together. This process was repeated for several different input drive amplitudes.

Simulated responses exhibited several properties that are qualitatively similar to experimental observations (Fig. S2b):

1) Responses exhibited oscillations in the gamma frequency range $(24,25)$. The increased power in the gamma frequency range was evident even for small input drives (e.g., $z=0.2$ ), well below the bifurcation ( $z \approx 0.5$; see Fig. 6).

2) Response amplitudes decreased with increasing frequency because of the lowpass filtering imposed by both the prefilter and the normalization circuit (Eq. S37). Response amplitudes decreased roughly proportional to frequency for frequencies greater than $10 \mathrm{~Hz}$, whereas it has been found experimentally to decrease with the square of frequency (26-28).

3) Response amplitudes decreased with increasing input drive at low frequencies (29).

4) Response amplitudes increased with input drive for a broad range of high frequencies above $30 \mathrm{~Hz}(26,30)$.

5) The oscillations were non-sinusoidal, sharp at the top and broad at the bottom of each cycle (Fig. S2a). This was also evident in the Fourier amplitude which exhibited harmonics (Fig. S2b), and in the asymmetric phase space trajectories (Fig 7d). Waveform shape may be an indicator to distinguish underlying mechanisms and pathophysiology (31). 
bioRxiv preprint doi: https://doi.org/10.1101/2020.03.22.002634; this version posted May 30, 2020. The copyright holder for this preprint (which was not certified by peer review) is the author/funder. All rights reserved. No reuse allowed without permission.

Dynamic Normalization SI Appendix

May 30, 2020

6) For simulations using the full model (Eqs. 1-6), the oscillatory activity was correlated across neurons with different orientation preferences at overlapping RF locations (32-34). The noise added to each neuron's input drive was statistically independent but, in spite of this, the phases of the oscillations tended to synchronize over time.

Note, however, that this process simulated the firing rates of individual principal cell, whereas local field potential (LFP), electrocorticography (ECoG), electroencephalogram (EEG), and magnetoencephalography (MEG) measurements depend on the synchronized membrane potential fluctuations across a large population of neurons $(28,35)$. 


\section{References}

1. D. J. Heeger, W. E. Mackey, Oscillatory recurrent gated neural integrator circuits (ORGaNICs), a unifying theoretical framework for neural dynamics. Proc Natl Acad Sci U S A 116, 22783-22794 (2019).

2. E. P. Simoncelli, W. T. Freeman, E. H. Adelson, D. J. Heeger, Shiftable multi-scale transforms. IEEE Transactions on Information Theory, Special Issue on Wavelets 38, 587-607 (1992).

3. D. J. Heeger, Half-squaring in responses of cat striate cells. Vis Neurosci 9, 427-443 (1992).

4. E. H. Adelson, J. R. Bergen, Spatiotemporal energy models for the perception of motion. Journal of the Optical Society of America A 2, 284-299 (1985).

5. J. C. Horton, W. F. Hoyt, The representation of the visual field in human striate cortex. Archives of Ophthalmology 109, 816-824 (1991).

6. S. O. Dumoulin, B. A. Wandell, Population receptive field estimates in human visual cortex. Neurolmage 39, 647-660 (2008).

7. J. Winawer, H. Horiguchi, R. A. Sayres, K. Amano, B. A. Wandell, Mapping hV4 and ventral occipital cortex: the venous eclipse. J Vis 10, 1 (2010).

8. J. Freeman, E. P. Simoncelli, Metamers of the ventral stream. Nat Neurosci 14, 1195-1201 (2011).

9. K. N. Kay, J. Winawer, A. Mezer, B. A. Wandell, Compressive spatial summation in human visual cortex. J Neurophysiol 110, 481-494 (2013).

10. Z. Liu, J. P. Gaska, L. D. Jacobson, D. A. Pollen, Interneuronal interaction between members of quadrature phase and anti-phase pairs in the cat's visual cortex. Vision Res 32, 1193-1198 (1992).

11. D. J. Heeger, Theory of cortical function. Proc Natl Acad Sci U S A 114, 1773-1782 (2017).

12. D. J. Heeger, W. E. Mackey, ORGaNICs: A Theory of Working Memory in Brains and Machines. arXiv preprint arXiv:1803.06288 (2018).

13. D. M. Wolpert, Z. Ghahramani, M. I. Jordan, An internal model for sensorimotor integration. Science 269, 1880-1882 (1995).

14. M. Kawato, Internal models for motor control and trajectory planning. Curr Opin Neurobiol 9, 718-727 (1999).

15. T. B. Crapse, M. A. Sommer, Corollary discharge across the animal kingdom. Nat Rev Neurosci 9, 587-600 (2008).

16. W. Bialek, I. Nemenman, N. Tishby, Predictability, complexity, and learning. Neural Computation 13, 2409-2463 (2001).

17. R. Nijhawan, Visual prediction: psychophysics and neurophysiology of compensation for time delays. Behav Brain Sci 31, 179-198; discussion 198-239 (2008).

18. S. E. Palmer, O. Marre, M. J. Berry, 2nd, W. Bialek, Predictive information in a sensory population. Proc Natl Acad Sci U S A 112, 6908-6913 (2015).

19. M. Carandini, D. J. Heeger, J. A. Movshon, Linearity and normalization in simple cells of the macaque primary visual cortex. J Neurosci 17, 8621-8644 (1997).

20. W. S. Geisler, D. G. Albrecht, Visual cortex neurons in monkeys and cats: detection, discrimination, and identification. Vis Neurosci 14, 897-919 (1997).

21. S. B.-D. Desmarchelier, A simple method to study local bifurcations of three and four-dimensional systems: characterizations and economic applications. (2017).

22. J. Guckenheimer, P. Holmes, "Local bifurcations" in Nonlinear oscillations, dynamical systems, and bifurcations of vector fields. (Springer, 1983), pp. 117-165. 
23. E. Doedel, P. Kamthan (2007) AUTO-07p: Continuation and bifurcation software for ordinary differential equations. (Concordia University, Montreal).

24. C. M. Gray, W. Singer, Stimulus-specific neuronal oscillations in orientation columns of cat visual cortex. Proc Natl Acad Sci U S A 86, 1698-1702. (1989).

25. X. Jia, D. Xing, A. Kohn, No consistent relationship between gamma power and peak frequency in macaque primary visual cortex. J Neurosci 33, 17-25 (2013).

26. J. A. Henrie, R. Shapley, LFP power spectra in V1 cortex: the graded effect of stimulus contrast. J Neurophysiol 94, 479-490 (2005).

27. K. J. Miller, L. B. Sorensen, J. G. Ojemann, M. den Nijs, Power-law scaling in the brain surface electric potential. PLoS Comput Biol 5, e1000609 (2009).

28. G. Buzsaki, C. A. Anastassiou, C. Koch, The origin of extracellular fields and currents--EEG, ECoG, LFP and spikes. Nat Rev Neurosci 13, 407-420 (2012).

29. B. Lima, M. M. Cardoso, Y. B. Sirotin, A. Das, Stimulus-related neuroimaging in task-engaged subjects is best predicted by concurrent spiking. J Neurosci 34, 13878-13891 (2014).

30. D. Hermes, K. J. Miller, B. A. Wandell, J. Winawer, Stimulus Dependence of Gamma Oscillations in Human Visual Cortex. Cereb Cortex 25, 2951-2959 (2015).

31. S. R. Cole, B. Voytek, Brain Oscillations and the Importance of Waveform Shape. Trends Cogn Sci 21, 137-149 (2017).

32. P. Berens, G. A. Keliris, A. S. Ecker, N. K. Logothetis, A. S. Tolias, Comparing the feature selectivity of the gamma-band of the local field potential and the underlying spiking activity in primate visual cortex. Front Syst Neurosci 2, 2 (2008).

33. X. Jia, M. A. Smith, A. Kohn, Stimulus selectivity and spatial coherence of gamma components of the local field potential. J Neurosci 31, 9390-9403 (2011).

34. E. Bartoli et al., Functionally Distinct Gamma Range Activity Revealed by Stimulus Tuning in Human Visual Cortex. Curr Biol 29, 3345-3358 e3347 (2019).

35. E. R. Kupers, N. C. Benson, J. Winawer, A visual encoding model links magnetoencephalography signals to neural synchrony in human cortex. bioRxiv 10.1101/2020.04.19.049197, 2020.2004.2019.049197 (2020). 